\title{
Compressions of Multiplication Operators and Their Characterizations
}

\author{
M. Cristina Câmara, Kamila Kliś-Garlicka, Bartosz Łanucha@, and \\ Marek Ptak (0)
}

\begin{abstract}
Dual truncated Toeplitz operators and other restrictions of the multiplication by the independent variable $M_{z}$ on the classical $L^{2}$ space on the unit circle are investigated. Commutators are calculated and commutativity is characterized. A necessary and sufficient condition for any operator to be a dual truncated Toeplitz operator is established. A formula for recovering its symbol is stated.
\end{abstract}

Mathematics Subject Classification. 47B32, 47B35, 30H10.

Keywords. Model space, truncated Toeplitz operator, dual truncated Toeplitz operator.

\section{Introduction}

Let $L^{2}:=L^{2}(\partial \mathbb{D})$ be the space of all measurable and square integrable functions on the unit circle $\mathbb{T}$ with respect to the normalized Lebesgue's measure. Recall that for $\varphi \in L^{\infty}$ the multiplication operator $M_{\varphi}$ is defined by $M_{\varphi} f=$ $\varphi f, f \in L^{2}$. Let $H^{2}$ denote the classical Hardy space and $H_{-}^{2}=L^{2} \ominus H^{2}$, let $P^{+}$ stand for the orthogonal projection from $L^{2}$ onto $H^{2}$ and let $P^{-}=I_{L^{2}}-P^{+}$ be the orthogonal projection from $L^{2}$ onto $H_{-}^{2}$. For $\varphi \in L^{\infty}$ recall the standard definitions

$$
T_{\varphi}=P^{+} M_{\varphi \mid H^{2}}, \quad H_{\varphi}=P^{-} M_{\varphi \mid H^{2}}
$$

The work of the first author was partially supported by FCT/Portugal through UID/MAT/04459/2020. The research of the second and the fourth authors was financed by the Ministry of Science and Higher Education of the Republic of Poland. 
The operators: $T_{\varphi} \in \mathscr{B}\left(H^{2}\right)$ and $H_{\varphi} \in \mathscr{B}\left(H^{2}, H_{-}^{2}\right)$ are called Toeplitz operator and Hankel operator (with symbol $\varphi$ ), respectively. We will use the notation $\mathscr{B}(\mathscr{H})$ or $\mathscr{B}(\mathscr{H}, \mathscr{K})$ for the set of all bounded operators on the Hilbert space $\mathscr{H}$ or from $\mathscr{H}$ to $\mathscr{K}$.

In what follows let $\theta$ denote a nonconstant inner function. Recall that the model space $K_{\theta}$ is defined as the orthogonal complement of $\theta H^{2}$ in $H^{2}$. One can therefore consider the decompositions

$$
H^{2}=K_{\theta} \oplus \theta H^{2} \quad \text { and } \quad L^{2}=K_{\theta} \oplus\left(K_{\theta}\right)^{\perp}=K_{\theta} \oplus \theta H^{2} \oplus H_{-}^{2} .
$$

According to the decomposition $L^{2}=K_{\theta} \oplus\left(K_{\theta}\right)^{\perp}$ the operator $M_{\varphi}, \varphi \in L^{\infty}$, can be written as

$$
M_{\varphi}=\left[\begin{array}{cc}
A_{\varphi}^{\theta} & \left(B_{\bar{\varphi}}^{\theta}\right)^{*} \\
B_{\varphi}^{\theta} & D_{\varphi}^{\theta}
\end{array}\right] .
$$

Although $M_{\varphi}$ is bounded if and only if $\varphi \in L^{\infty}$, some restrictions of $M_{\varphi}$ may still be bounded even if they do not have a bounded symbol (for example, for $A_{\varphi}^{\theta}$ see [1] and for $B_{\varphi}^{\theta}$ see Remark 4). Hence, now let $\varphi \in L^{2}$ and set the densely defined multiplication operator $M_{\varphi}: D\left(M_{\varphi}\right) \rightarrow L^{2}$ as $M_{\varphi} f=$ $\varphi f$, where $D\left(M_{\varphi}\right)=\left\{f \in L^{2}: \varphi f \in L^{2}\right\}$. Note that $L^{\infty} \subset D\left(M_{\varphi}\right)$ for all $\varphi \in L^{2}$. Let $P_{\theta}$ denote the orthogonal projection from $L^{2}$ onto $K_{\theta}$ and let $P_{\theta}^{\perp}=I_{L^{2}}-P_{\theta}$ be the orthogonal projection from $L^{2}$ onto $\left(K_{\theta}\right)^{\perp}$. Recall after [11] that $K_{\theta}^{\infty}:=K_{\theta} \cap L^{\infty}$ is a dense subset of $K_{\theta}$. Since $\bar{z} \overline{H^{\infty}}$ is a dense subset of $H_{-}^{2}$ and $\theta H^{\infty}$ is a dense subset of $\theta H^{2}$, it follows that $K_{\theta}^{\perp} \cap L^{\infty}$ is a dense subset of $K_{\theta}^{\perp}$. We define

$$
A_{\varphi}^{\theta}=P_{\theta} M_{\varphi \mid K_{\theta} \cap L^{\infty}}, \quad B_{\varphi}^{\theta}=P_{\theta}^{\perp} M_{\varphi \mid K_{\theta} \cap L^{\infty}} \quad \text { and } \quad D_{\varphi}^{\theta}=P_{\theta}^{\perp} M_{\varphi \mid K_{\theta}^{\perp} \cap L^{\infty}} .
$$

If $A_{\varphi}^{\theta}$ extends to the whole $K_{\theta}$ as a bounded operator, it is called a truncated Toeplitz operator (TTO). Similarly, if $B_{\varphi}^{\theta}$ extends to a bounded operator from $K_{\theta}$ to $K_{\theta}^{\perp}$, it is called a big truncated Hankel operator (THO) (see [17]), and if $D_{\varphi}^{\theta}$ extends to the whole $K_{\theta}^{\perp}$ as a bounded operator, it is called a dual truncated Toeplitz operator (DTTO). Let us fix the notation

$$
\begin{aligned}
\mathscr{T}\left(K_{\theta}\right) & =\left\{A_{\varphi}^{\theta}: \varphi \in L^{2} \text { and } A_{\varphi}^{\theta} \text { is bounded }\right\}, \\
\mathscr{T}\left(K_{\theta}, K_{\theta}^{\perp}\right) & =\left\{B_{\varphi}^{\theta}: \varphi \in L^{2} \text { and } B_{\varphi}^{\theta} \text { is bounded }\right\}, \\
\mathscr{T}\left(K_{\theta}^{\perp}\right) & =\left\{D_{\varphi}^{\theta}: \varphi \in L^{2} \text { and } D_{\varphi}^{\theta} \text { is bounded }\right\} .
\end{aligned}
$$

Model spaces, which provide the natural setting for truncated Toeplitz operators, have generated enormous interest and they are relevant in connection with a variety of topics such as the Schrödinger operator, classical extremal problems in control theory, Hankel operators and Toeplitz matrices (see for instance [13] and [11]). Natural conjugations, which model spaces and the whole $L^{2}$ possess (see [5]), make model spaces even more natural in the context of pysics [12]. Their orthogonal complements in $L^{2}$ also appear in numerous applications. In the equivalent setting of the real line $[9,16]$, using time and 
frequency as the natural variables, and taking the inner function $\theta=\theta_{\lambda}$ with $\theta_{\lambda}(\xi)=\exp (i \lambda \xi)$ for $\xi \in \mathbb{R}$, they appear via the Fourier transform, for instance, as high frequency signals, which are of decisive importance in electronics, or as outputs of high-pass filters. Dual truncated Toeplitz operators, acting on these spaces have realizations, for example, in long distance communication links with several regenerators along the path that cancel low-frequency noise using high-pass filters, or in the description of wave propagation in the presence of finite-length obstacles.

Systematic study of truncated Toeplitz operators $A_{\varphi}^{\theta}$ (for general $\varphi \in L^{2}$ ) was started in [22] while the properties of dual truncated Toeplitz operators $D_{\varphi}^{\theta}$ were investigated in $[8,15,20]$ and more recently in $[2,6,7,21]$. Truncated Hankel operators were studied in [17] and also in [14], but there is a different definition of $B_{\varphi}^{\theta}$ (see also [3]).

The main purpose of this paper is to advance the study of dual truncated Toeplitz operators $\mathscr{T}\left(K_{\theta}^{\perp}\right)$ and big truncated Hankel operators $\mathscr{T}\left(K_{\theta}, K_{\theta}^{\perp}\right)$. The first result worth mentioning is a full characterization of rank-one elements in $\mathscr{T}\left(K_{\theta}, K_{\theta}^{\perp}\right)$ with $L^{\infty}$ symbols - Sect. 3. Note that it is clear from [8, Property 2.2] that there are no rank-one operators in $\mathscr{T}\left(K_{\theta}^{\perp}\right)$.

The property of commutativity with the operator of multiplication by the independent variable is very important. In the classical situation, in the space $L^{2}$, only multiplication operators $M_{\varphi}$ with $\varphi \in L^{\infty}$ commute with $M_{z}$. In the case of the Hardy space $H^{2}$, the operators which commute with the Toeplitz operator $T_{z}$ (called the unilateral shift) are Toeplitz operators $T_{\varphi}$ with $\varphi$ being a bounded analytic symbol $\left(\varphi \in H^{\infty}\right)$. In the case of a model space $K_{\theta}$, where $\theta$ is a nonconstant inner function, it is shown in [23] that an operator commuting with the compression of the unilateral shift to the model space $P_{\theta} T_{z \mid K_{\theta}}=A_{z}^{\theta}$ has to be a truncated Toeplitz operator with an analytic symbol. In Sect. 4 we discuss similar questions for dual truncated Toeplitz operators.

On the other hand, it is known that a bounded linear operator $T \in \mathscr{B}\left(H^{2}\right)$ is a Toeplitz operator if and only if $T=\left(T_{z}\right)^{*} T T_{z}$. Similar characterizations (in terms of compressions of $M_{z}$ ) are known for Hankel operators and dual Toeplitz operators. In [22] D. Sarason characterized bounded truncated Toeplitz operators in terms of the compressions of $M_{z}$ to $K_{\theta}$. For example, he characterized truncated Toeplitz operators expressing the difference $A-A_{z}^{\theta} A A_{\bar{z}}^{\theta}$ as a specific rank-two operator. In Sect. 5 a relation similar to the one above is given for dual truncated Toeplitz operators. For any $D \in \mathscr{T}\left(K_{\theta}^{\perp}\right)$ we also express $D-D_{z}^{\theta} D\left(D_{z}^{\theta}\right)^{*}$ by rank-two operators. However, it does not lead to a characterization of DTTO. To obtain such a characterization we use specific restrictions of dual truncated Toeplitz operators in Sect. 6. Finally, in Sect. 7, Theorem 27 gives a necessary and sufficient condition for any operator $D \in \mathscr{B}\left(K_{\theta}^{\perp}\right)$ to be a DTTO. Moreover, using the formula given there we can easily recover its unique symbol. 


\section{Basic Properties}

For any Hilbert space $\mathscr{H}$ and $h, g \in \mathscr{H}$ we define the rank-one operator $h \otimes g$ as $h \otimes g(f)=\langle f, g\rangle h$. Some rank-one operators are the commutators in $\mathscr{B}\left(L^{2}\right)$.

Lemma 1. Let $M_{z}$ be the operator of multiplication by the independent variable in $L^{2}$. Then

(1) $P^{+} M_{z}-M_{z} P^{+}=1 \otimes \bar{z}$,

(2) $P^{-} M_{\bar{z}}-M_{\bar{z}} P^{-}=\bar{z} \otimes 1$.

\subsection{Conjugation}

There is a natural conjugation (an antiunitary involution) connected with a model space (see for instance [5,12]). For an inner function $\theta$ define $C_{\theta}: L^{2} \rightarrow$ $L^{2}$ by

$$
C_{\theta} f(z)=\theta(z) \overline{z f(z)}, \quad|z|=1 .
$$

Then $C_{\theta}$ is an antilinear isometric involution on $L^{2}$, which implies that $\left\langle C_{\theta} f\right.$, $\left.C_{\theta} g\right\rangle=\langle g, f\rangle$ for $f, g \in L^{2}$. One can easily verify that

$$
C_{\theta} M_{\varphi} C_{\theta}=M_{\bar{\varphi}} .
$$

It is well known ([12]) that $C_{\theta}$ preserves $K_{\theta}$. Moreover, $C_{\theta}\left(\theta H^{2}\right)=H_{-}^{2}$ and $C_{\theta}\left(H_{-}^{2}\right)=\theta H^{2}$, so $C_{\theta}$ also preserves $\left(K_{\theta}\right)^{\perp}$. Hence,

$$
C_{\theta}=\left[\begin{array}{cc}
C_{\theta \mid K_{\theta}} & 0 \\
0 & C_{\theta \mid\left(K_{\theta}\right)^{\perp}}
\end{array}\right] .
$$

Corollary 2. Let $\varphi \in L^{2}$.

(1) If $A_{\varphi}^{\theta} \in \mathscr{B}\left(K_{\theta}\right)$, then $C_{\theta} A_{\varphi}^{\theta} C_{\theta}=A_{\bar{\varphi}}^{\theta}$.

(2) If $B_{\varphi}^{\theta} \in \mathscr{B}\left(K_{\theta}, K_{\theta}^{\perp}\right)$, then $B_{\bar{\varphi}}^{\theta} \in \mathscr{B}\left(K_{\theta}, K_{\theta}^{\perp}\right)$ and $C_{\theta} B_{\varphi}^{\theta} C_{\theta}=B_{\bar{\varphi}}^{\theta}$.

(3) If $D_{\varphi}^{\theta} \in \mathscr{B}\left(K_{\theta}^{\perp}\right)$, then $C_{\theta} D_{\varphi}^{\theta} C_{\theta}=D_{\bar{\varphi}}^{\theta}$.

Proof. Using the representation (1.1) and (2.2) on $\left(K_{\theta} \cap L^{\infty}\right) \oplus\left(K_{\theta}^{\perp} \cap L^{\infty}\right)$ the equality below holds:

$$
\begin{aligned}
{\left[\begin{array}{cc}
A_{\bar{\varphi}}^{\theta}\left(B_{\varphi}^{\theta}\right)^{*} \\
B_{\bar{\varphi}}^{\theta} & D_{\bar{\varphi}}^{\theta}
\end{array}\right] } & =\left[\begin{array}{cc}
C_{\theta \mid K_{\theta}} & 0 \\
0 & C_{\theta \mid\left(K_{\theta}\right)^{\perp}}
\end{array}\right] \cdot\left[\begin{array}{cc}
A_{\varphi}^{\theta}\left(B_{\varphi}^{\theta}\right)^{*} \\
B_{\varphi}^{\theta} & D_{\varphi}^{\theta}
\end{array}\right] \cdot\left[\begin{array}{cc}
C_{\theta \mid K_{\theta}} & 0 \\
0 & C_{\theta \mid\left(K_{\theta}\right)^{\perp}}
\end{array}\right] \\
& =\left[\begin{array}{cc}
C_{\theta} A_{\varphi}^{\theta} C_{\theta} & C_{\theta}\left(B_{\bar{\varphi}}^{\theta}\right)^{*} C_{\theta} \\
C_{\theta} B_{\varphi}^{\theta} C_{\theta} & C_{\theta} D_{\varphi}^{\theta} C_{\theta}
\end{array}\right] .
\end{aligned}
$$

In particular $C_{\theta} B_{\varphi}^{\theta} C_{\theta}=B_{\bar{\varphi}}^{\theta}$ on $K_{\theta} \cap L^{\infty}$. Since $B_{\varphi}^{\theta}$ is bounded, $B_{\bar{\varphi}}^{\theta}$ is bounded, too, and the equality holds on $K_{\theta}$. The other equalities can be shown similarly.

Part (1) of Corollary 2 was proved by D. Sarason [22] while part (3) was shown in [15]. 


\subsection{Zero Operators}

Recall that $k_{w}=\frac{1}{1-\bar{w} z}$ is a reproducing kernel for all functions $f \in H^{2}$, i.e., $f(w)=\left\langle f, k_{w}\right\rangle$ for $w \in \mathbb{D}$. Let $\theta$ be a nonconstant inner function. Then $k_{w}^{\theta}=P_{\theta} k_{w}=(1-\overline{\theta(w)} \theta) k_{w}$ is a reproducing kernel for all functions $f \in K_{\theta}$, i.e., $f(w)=\left\langle f, k_{w}^{\theta}\right\rangle$ for $w \in \mathbb{D}$. Denote $\tilde{k}_{w}^{\theta}=C_{\theta} k_{w}^{\theta}, \tilde{k}_{w}^{\theta}(z)=\frac{\theta(z)-\theta(w)}{z-w}$. Note that $C_{\theta} f(w)=\overline{\left\langle f, \tilde{k}_{w}^{\theta}\right\rangle}$ for $w \in \mathbb{D}$.

It is well known that a Toeplitz operator is uniquely determined by its symbol, that is, $T_{\varphi}=0$ if and only if $\varphi=0$. The same is true for dual Toeplitz operators, but is not for Hankel operators.

As for truncated Toeplitz operators, it was proved by D. Sarason in [22] that the symbol of a truncated Toeplitz operator is not uniquely determined. A similar result for truncated Hankel operators was obtained by P. Ma, F. Yan and Zhang in D. [17]. On the other hand, X. Ding and Y. Sang showed in [8] that the symbol of a dual truncated Toeplitz operator is unique. Summing up, we have the following.

Proposition 3. Let $\theta$ be a nonconstant inner function and let $\varphi \in L^{2}$. Then

(1) $A_{\varphi}^{\theta}=0$ if and only if $\varphi \in \theta H^{2}+\overline{\theta H^{2}}$;

(2) $B_{\varphi}^{\theta}=0$ if and only if $\varphi$ is a constant function;

(3) $D_{\varphi}^{\theta}=0$ if and only if $\varphi=0$.

The proof of part (2) given in [17] was based on the fact that $B_{\varphi}^{\theta}$ can be expressed as a block operator built from certain products of classical Hankel operators, and only for $\varphi \in L^{\infty}$. But (2) can be proved in an alternative, simpler way. Namely, note that $B_{\varphi}^{\theta}=0$ means that $P_{\theta}^{\perp}(\varphi f)=0$ for all $f \in K_{\theta}$. The latter happens if and only if $\varphi f \in K_{\theta}$ for all $f \in K_{\theta}$, which means that the multiplication operator $M_{\varphi}$ preserves $K_{\theta}$. By [10, Proposition 2.2], this is equivalent to $\varphi$ being a constant function.

\subsection{Boundedness}

It is a classical result that $M_{\varphi}$ is bounded on $L^{2}$ if and only if $\varphi \in L^{\infty}$. It was proved in [8] that a dual truncated Toeplitz operator is bounded if and only if its symbol is bounded. Hence

$$
\mathscr{T}\left(K_{\theta}^{\perp}\right)=\left\{D_{\varphi}^{\theta}: \varphi \in L^{\infty}\right\} .
$$

On the other hand, it is known that there exist bounded truncated Toeplitz operators without bounded symbols [1].

Remark 4. The latter is true also for truncated Hankel operators. To see this it is enough to consider a finite dimensional model space $K_{\theta}(\theta$ is a finite Blaschke product). Then $B_{\varphi}^{\theta}$ is clearly bounded for every $\varphi \in L^{2}$. But if $B_{\varphi}^{\theta}=B_{\chi}^{\theta}$ for some $\chi \in L^{\infty}$, then by Proposition 3 there exists $c \in \mathbb{C}$ such that $\varphi-\chi=c$ and so $\varphi \in L^{\infty}$. 
Let $\varphi \in L^{2}$ and consider the matrix

$$
M_{\varphi}=\left[\begin{array}{cc}
A_{\varphi}^{\theta} & \left(B_{\bar{\varphi}}^{\theta}\right)^{*} \\
B_{\varphi}^{\theta} & D_{\varphi}^{\theta}
\end{array}\right] .
$$

Corollary 2 shows that $B_{\varphi}^{\theta}$ is bounded if and only if $\left(B_{\bar{\varphi}}^{\theta}\right)^{*}$ is bounded. Note also that if $\varphi \notin L^{\infty}$ even if $A_{\varphi}^{\theta}, B_{\varphi}^{\theta}$ are bounded $M_{\varphi}$ is not bounded since $D_{\varphi}^{\theta}$ is not bounded.

\section{Rank-One and Defect Operators}

In [22] D. Sarason described all rank-one operators in $\mathscr{T}\left(K_{\theta}\right)$. Here we completely characterize rank-one operators in $\mathscr{T}\left(K_{\theta}, K_{\theta}^{\perp}\right)$.

Let $f$ be analytic on some open set $G$. Denote the $n$-th Taylor polynomial of $f$ at $a \in G$ as

$$
\mathbf{P}_{n}(f, a)(z)=f(a)+\frac{1}{1 !} f^{\prime}(a)(z-a)+\cdots+\frac{1}{(n-1) !} f^{(n-1)}(a)(z-a)^{n-1} .
$$

Let now $f \in H_{-}^{2}$. Then $f_{+}=\bar{z} \bar{f} \in H^{2}$ and it is a standard procedure to define the analytic extension of $f$ to $\mathbb{D}^{e}=\mathbb{C} \backslash \overline{\mathbb{D}}$ as $f(z)=\frac{1}{z} \overline{f_{+}\left(\bar{z}^{-1}\right)}$ for $z \in \mathbb{D}^{e}$. If $f \in H_{-}^{2}$ and $b \in \mathbb{D}^{e}$, then it is not hard to see that $\frac{f(\cdot)-f(b)}{z-b} \in H_{-}^{2}$. Moreover, using the formula $\lim _{z \rightarrow b}\left(f(z)-\mathbf{P}_{n}(f, b)(z)\right)(z-b)^{-n}=\frac{1}{n !} f^{(n)}(b)$, we have $\frac{f(\cdot)-\mathbf{P}_{n}(f, b)}{(z-b)^{n}} \in H_{-}^{2}$. In particular, we apply this below to $\bar{\theta} f \in H_{-}^{2}$ for $f \in K_{\theta}$.

Lemma 5. Let $\theta$ be a nonconstant inner function and $n \in \mathbb{N}$. If $f \in K_{\theta}$, then

(1) $P_{\theta}^{\perp}\left(z^{n} f\right)=\theta z^{n-1} \overline{\mathbf{P}_{n}\left(C_{\theta} f, 0\right)}$;

(2) if $a \in \mathbb{D}$ then $P_{\theta}^{\perp}\left((z-a)^{-n} f\right)=(z-a)^{-n} \mathbf{P}_{n}(f, a)$;

(3) if $b \in \mathbb{D}^{e}$ then $P_{\theta}^{\perp}\left((z-b)^{-n} f\right)=\theta(z-b)^{-n} \mathbf{P}_{n}(\bar{\theta} f, b)$.

Proof. To see (1) note that $z^{n} f \in H^{2}$ and thus

$$
P_{\theta}^{\perp}\left(z^{n} f\right)=\theta P^{+} \bar{\theta} z^{n} f=\theta P^{+} z^{n-1} \overline{C_{\theta} f}=\theta z^{n-1} \overline{\mathbf{P}_{n}\left(C_{\theta} f, 0\right)} .
$$

For (2) note that $\bar{\theta}(z-a)^{-n} f \in H_{-}^{2}$. Hence

$$
\begin{aligned}
P_{\theta}^{\perp}\left((z-a)^{-n} f\right) & =\left(P^{-}+\theta P^{+} \bar{\theta}\right)\left((z-a)^{-n} f\right) \\
& =P^{-}\left((z-a)^{-n} f\right)=(z-a)^{-n} \mathbf{P}_{n}(f, a) .
\end{aligned}
$$

To observe (3) note that $(z-b)^{-n} f \in H^{2}$, so

$$
\begin{aligned}
P_{\theta}^{\perp}\left((z-b)^{-n} f\right)= & \theta P^{+}\left((z-b)^{-n} \bar{\theta} f\right) \\
= & \theta P^{+}\left((z-b)^{-n}\left(\bar{\theta} f-\mathbf{P}_{n}(\bar{\theta} f, b)\right)\right) \\
& +\theta P^{+}\left((z-b)^{-n} \mathbf{P}_{n}(\bar{\theta} f, b)\right) \\
= & \theta(z-b)^{-n} \mathbf{P}_{n}(\bar{\theta} f, b) .
\end{aligned}
$$


Before we state the result recall that $\operatorname{dim} K_{\theta}=1$ if and only if $\theta(z)=$ $\lambda \frac{a-z}{1-\bar{a} z}$ with $\lambda \in \mathbb{T}, a \in \mathbb{D}$, in particular for $\theta(z)=z$.

Theorem 6. Let $\theta$ be a nonconstant inner function.

(1) If $\operatorname{dim} K_{\theta}=1$, then all nonzero operators from $\mathscr{T}\left(K_{\theta}, K_{\theta}^{\perp}\right)$ are of rank one.

(2) Assume that $\operatorname{dim} K_{\theta}>1$. If $B_{\varphi}^{\theta} \in \mathscr{T}\left(K_{\theta}, K_{\theta}^{\perp}\right)$ with $\varphi \in L^{\infty}$ is of rank one, then it is a constant multiple of one of the operators below

$B_{z k_{w}}^{\theta}=\theta k_{w} \otimes \tilde{k}_{w}^{\theta}, \quad$ or $\quad B_{\bar{z} \bar{k}_{w}}^{\theta}=\bar{z} \bar{k}_{w} \otimes k_{w}^{\theta}, \quad$ where $\quad w \in \mathbb{D}$.

Proof. Let $B_{\varphi}^{\theta}$ be a rank-one operator and let $\psi \in K_{\theta}^{\perp}$ span the range of $B_{\varphi}^{\theta}$. For $\theta$ such that $\operatorname{dim} K_{\theta}>1$, there exists $f_{\theta} \in K_{\theta}$ with $f_{\theta}(0)=0$, so $\bar{z} f_{\theta} \in K_{\theta}$. Then $P_{\theta}^{\perp}\left(\varphi f_{\theta}\right)=\lambda_{1} \psi$ and $P_{\theta}^{\perp}\left(\varphi \bar{z} f_{\theta}\right)=\lambda_{2} \psi$ for some $\lambda_{1}, \lambda_{2} \in \mathbb{C}$. Thus $\lambda_{2} P_{\theta}^{\perp}\left(\varphi f_{\theta}\right)-\lambda_{1} P_{\theta}^{\perp}\left(\varphi \bar{z} f_{\theta}\right)=0$ and hence $\left(\lambda_{2}-\lambda_{1} \bar{z}\right) \varphi f_{\theta}=g_{\theta} \in K_{\theta}$.

If $\theta$ is a finite Blaschke product, then $f_{\theta}$ and $g_{\theta}$ are rational, and it follows that $\varphi$ is also rational, without poles on the unit circle $\mathbb{T}$, because $\varphi \in L^{\infty}$. If $\theta$ is not a finite Blaschke product, it follows from [17, Theorem 1.2] that $\varphi$ is also a rational function without poles on $\mathbb{T}$. Therefore $\varphi$ is a linear combination of functions of the form $z^{n},(z-a)^{-n}$ with $a \in \mathbb{D},(z-b)^{-n}$ with $b \in \mathbb{D}^{e}, n \in \mathbb{N}$.

In view of Lemma 5 we see that $B_{\varphi}^{\theta}$ can have rank one if and only if $n=1$, i.e., $\varphi=z, \varphi=(z-a)^{-1}, a \in \mathbb{D}$ or $\varphi=(z-b)^{-1}, b \in \mathbb{D}^{e}$. We will use the Taylor expansion with $n=1$. Let $f \in K_{\theta}$ and consider the case $\varphi=z$. Then

$$
B_{z}^{\theta} f=B_{z k_{0}}^{\theta} f=\theta \overline{C_{\theta} f(0)}=\left\langle f, \tilde{k}_{0}^{\theta}\right\rangle \theta=\left(\theta \otimes \tilde{k}_{0}^{\theta}\right) f .
$$

Set $a=w$, and consider $\varphi=(z-w)^{-1}=\bar{z}(1-w \bar{z})^{-1}=\bar{z} \bar{k}_{w}$. Then

$$
\begin{aligned}
B_{\bar{z} \bar{k}_{w}}^{\theta} f & =f(w)(z-w)^{-1}=\left\langle f, k_{w}^{\theta}\right\rangle(z-w)^{-1}=\left((z-w)^{-1} \otimes k_{w}^{\theta}\right) f \\
& =\left(\bar{z} \bar{k}_{w} \otimes k_{w}^{\theta}\right) f .
\end{aligned}
$$

Now the last case is $\varphi=(z-b)^{-1}, b \in \mathbb{D}^{e}$. Let us set $w=\bar{b}^{-1}(w \neq 0)$. Then using the formula for the analytic extension we have $(\bar{\theta} f)(b)=\bar{w} \overline{C_{\theta} f(w)}$. Since $z k_{w}$ and $-\bar{w}^{-2}\left(z-\frac{1}{\bar{w}}\right)^{-1}$ differ only by a constant, thus, by Lemma 5 (3) we have

$$
\begin{aligned}
B_{z k_{w}}^{\theta} f & =-\bar{w}^{-2} B_{\left(z-\frac{1}{\bar{w}}\right)^{-1}}^{\theta} f=-\bar{w}^{-2} \theta\left(z-\frac{1}{\bar{w}}\right)^{-1}(\bar{\theta} f)\left(\frac{1}{\bar{w}}\right) \\
& =\theta(1-\bar{w} z)^{-1} \overline{\left(C_{\theta} f\right)(w)}=\left(\theta k_{w} \otimes \tilde{k}_{w}^{\theta}\right) f .
\end{aligned}
$$

The following is a consequence of Theorem 6 .

Corollary 7. (1) $B_{z}^{\theta}=\theta \otimes \widetilde{k}_{0}^{\theta}$;

(2) $\left(B_{z}^{\theta}\right)^{*}=\widetilde{k}_{0}^{\theta} \otimes \theta$;

(3) $B_{\bar{z}}^{\theta}=\bar{z} \otimes k_{0}^{\theta}$;

(4) $\left(B_{\bar{z}}^{\theta}\right)^{*}=k_{0}^{\theta} \otimes \bar{z}$. 
Recall from [22] that for truncated Toeplitz operators we have the following formulas for the defect operators:

$$
A_{z}^{\theta} A_{\bar{z}}^{\theta}=I_{K_{\theta}}-k_{0}^{\theta} \otimes k_{0}^{\theta} \quad \text { and } \quad A_{\bar{z}}^{\theta} A_{z}^{\theta}=I_{K_{\theta}}-\tilde{k}_{0}^{\theta} \otimes \tilde{k}_{0}^{\theta} .
$$

Similarly, we have the following.

Proposition 8. Let $\theta$ be a nonconstant inner function. Then

(1) $D_{z}^{\theta} D_{\bar{z}}^{\theta}=I_{K_{\theta}^{\perp}}-\left(1-|\theta(0)|^{2}\right) \theta \otimes \theta$;

(2) $D_{\bar{z}}^{\theta} D_{z}^{\theta}=I_{K_{\theta}^{\perp}}-\left(1-|\theta(0)|^{2}\right) \bar{z} \otimes \bar{z}$;

(3) $B_{z}^{\theta}\left(B_{\bar{z}}^{\theta}\right)^{*}=\overline{\theta^{\prime}(0)} \theta \otimes \bar{z}$;

(4) $\left(B_{\bar{z}}^{\theta}\right)^{*} B_{z}^{\theta}=0$.

Proof. Note that for $f, g \in K_{\theta}^{\perp}$ we have

$$
\begin{aligned}
\left\langle D_{z}^{\theta} D_{\bar{z}}^{\theta} f, g\right\rangle & =\left\langle z\left(\bar{z} f-P_{\theta}(\bar{z} f)\right), g\right\rangle \\
& =\left\langle f-z P_{\theta}(\bar{z} f), g\right\rangle=\langle f, g\rangle-\left\langle z P_{\theta}(\bar{z} f), g\right\rangle .
\end{aligned}
$$

Since

$$
\begin{aligned}
P_{\theta}(\bar{z} f) & =P^{+} \theta P^{-} \bar{\theta}\left(\bar{z} P^{-} f+\bar{z} P_{\theta H^{2}} f\right) \\
& =P^{+} \theta P^{-} \bar{\theta} \bar{z} P_{\theta H^{2}} f=\langle\bar{\theta} f, 1\rangle P^{+} \theta \bar{z}=\langle f, \theta\rangle \tilde{k}_{0}^{\theta},
\end{aligned}
$$

we have

$$
\left\langle D_{z}^{\theta} D_{\bar{z}}^{\theta} f, g\right\rangle=\langle f, g\rangle-\langle f, \theta\rangle\left\langle z \tilde{k}_{0}^{\theta}, g\right\rangle=\langle f, g\rangle-\langle f, \theta\rangle\left\langle P_{\theta}^{\perp}\left(z \tilde{k}_{0}^{\theta}\right), g\right\rangle .
$$

Note that $P_{\theta}^{\perp}\left(z \tilde{k}_{0}^{\theta}\right)=\left(1-|\theta(0)|^{2}\right) \theta$. Hence

$$
\begin{aligned}
\left\langle D_{z}^{\theta} D_{\bar{z}}^{\theta} f, g\right\rangle & =\langle f, g\rangle-\left\langle\langle f, \theta\rangle\left(1-|\theta(0)|^{2}\right) \theta, g\right\rangle \\
& =\left\langle\left(I_{\left(K_{\theta}\right)^{\perp}}-\left(1-|\theta(0)|^{2}\right) \theta \otimes \theta\right) f, g\right\rangle .
\end{aligned}
$$

To prove (2) note that, by Corollary 2 ,

$$
\begin{aligned}
D_{\bar{z}}^{\theta} D_{z}^{\theta} & =C_{\theta} D_{z}^{\theta} D_{\bar{z}}^{\theta} C_{\theta}=C_{\theta}\left(I_{\left(K_{\theta}\right)^{\perp}}-\left(1-|\theta(0)|^{2}\right) \theta \otimes \theta\right) C_{\theta} \\
& =I_{\left(K_{\theta}\right)^{\perp}}-\left(1-|\theta(0)|^{2}\right) C_{\theta} \theta \otimes C_{\theta} \theta=I_{K_{\theta}^{\perp}}-\left(1-|\theta(0)|^{2}\right) \bar{z} \otimes \bar{z} .
\end{aligned}
$$

Calculating (3) we will use Corollary 7 and the formula for multiplication of rank-one operators (see [19])

$$
B_{z}^{\theta}\left(B_{\bar{z}}^{\theta}\right)^{*}=\left(\theta \otimes \widetilde{k}_{0}^{\theta}\right)\left(k_{0}^{\theta} \otimes \bar{z}\right)=\left\langle k_{0}^{\theta}, \tilde{k}_{0}^{\theta}\right\rangle \theta \otimes \bar{z}=\overline{\theta^{\prime}(0)} \theta \otimes \bar{z} .
$$

We get the last formula similarly.

\section{Commutativity with Restrictions of $M_{z}$}

Starting the study of commutativity with $D_{z}^{\theta}=P_{\theta}^{\perp} M_{z \mid K_{\theta}^{\perp}}$ we first give an example of an operator which commutes with $D_{z}^{\theta}$ and is not a dual truncated Toeplitz operator. 
Example 9. Let $\theta$ be an inner function such that $\theta(0)=0$. For $\varphi \in H^{\infty} \backslash\{0\}$ define $D \in \mathscr{B}\left(\theta H^{2} \oplus H_{-}^{2}\right)$ by $D=P_{\theta H^{2}} M_{\varphi \mid \theta H^{2}} \oplus 0$. Then for any $f, g \in H^{2}$ we have

$$
D_{z}^{\theta} D(\theta f+\bar{z} \bar{g})=D_{z}^{\theta}(\varphi \theta f)=z \varphi \theta f .
$$

On the other hand,

$$
\begin{aligned}
D_{z}^{\theta}(\theta f+\bar{z} \bar{g}) & =P_{\theta}^{\perp}(z \theta f+\bar{g})=z \theta f+\overline{g-g(0)}+P_{\theta}^{\perp}(\overline{g(0)}) \\
& =z \theta f+\overline{g-g(0)}+\overline{g(0)} \theta \overline{\theta(0)}=z \theta f+\overline{g-g(0)}
\end{aligned}
$$

since $\theta(0)=0$. It follows that

$$
D D_{z}^{\theta}(\theta f+\bar{z} \bar{g})=D(z \theta f+\overline{g-g(0)})=z \varphi \theta f
$$

and so $D$ commutes with $D_{z}^{\theta}$. To see that $D \notin \mathscr{T}\left(K_{\theta}^{\perp}\right)$ observe that $D$ does not satisfy condition (c) from Corollary 2, that is, $C_{\theta} D C_{\theta} \neq D^{*}$. Indeed, here $D^{*}=P_{\theta H^{2}} M_{\bar{\varphi} \mid \theta H^{2}} \oplus 0$, while

$$
C_{\theta} D C_{\theta}(\theta f+\bar{z} \bar{g})=C_{\theta} D(\bar{z} \bar{f}+\theta g)=C_{\theta}(\varphi \theta g)=\overline{\varphi z g},
$$

which means that $C_{\theta} D C_{\theta}=0 \oplus P^{-} M_{\bar{\varphi} \mid H_{-}^{2}}$.

Below we calculate the commutator of a given dual truncated Toeplitz operator with $D_{z}^{\theta}$. It shows how far is this relation from commutativity. Let us start with the following lemma.

Lemma 10. Let $\varphi \in L^{2}, \varphi=\bar{z} \overline{\left(\chi_{1}+\theta \chi_{2}\right)}+\psi_{1}+\theta \psi_{2}$ with $\chi_{1}, \psi_{1} \in K_{\theta}, \chi_{2}, \psi_{2} \in$ $H^{2}$ according to the decomposition $L^{2}=\bar{z} \overline{\left(K_{\theta} \oplus \theta H^{2}\right)} \oplus K_{\theta} \oplus \theta H^{2}$. Then

(1) $B_{\varphi}^{\theta} k_{0}^{\theta}=\varphi k_{0}^{\theta}-P_{\theta} \varphi+\overline{\theta(0)} C_{\theta} P_{\theta}(\bar{z} \bar{\varphi})$;

(2) $B_{\bar{\varphi}}^{\theta} \tilde{k}_{0}^{\theta}=C_{\theta} B_{\varphi}^{\theta} k_{0}^{\theta}=C_{\theta} P_{\theta}^{\perp}\left(\varphi k_{0}^{\theta}\right)$;

(3) $\left(B_{\bar{\varphi}}^{\theta}\right)^{*} \theta=P_{\theta}(\theta \varphi)=C_{\theta} \chi_{1}$;

(4) $\left(B_{\varphi}^{\theta}\right)^{*} \bar{z}=C_{\theta}\left(B_{\bar{\varphi}}^{\theta}\right)^{*} \theta=\chi_{1}=C_{\theta} P_{\theta}(\theta \varphi)$.

Proof. Note that

$$
\begin{aligned}
B_{\varphi}^{\theta} k_{0}^{\theta} & =P_{\theta}^{\perp}\left(\varphi k_{0}^{\theta}\right)=P_{\theta}^{\perp} \varphi(1-\overline{\theta(0)} \theta) \\
& =\varphi-P_{\theta} \varphi-\overline{\theta(0)} P_{\theta}^{\perp}\left(\theta \bar{z} \bar{\chi}_{1}+\bar{z} \bar{\chi}_{2}+\theta \psi_{1}+\theta^{2} \psi_{2}\right) \\
& =\varphi-\psi_{1}-\overline{\theta(0)}\left(\theta \varphi-C_{\theta} \chi_{1}\right)=\varphi k_{0}^{\theta}-\psi_{1}+\overline{\theta(0)} C_{\theta} \chi_{1} .
\end{aligned}
$$

The second condition follows from Corollary 2. Condition (3) is straightforward, whereas (4) follows from (3) and Corollary 2.

The next theorem gives the formulas for the commutators.

Theorem 11. Let $\varphi \in L^{2}$. Then

(1) if $D_{\varphi}^{\theta} \in \mathscr{B}\left(K_{\theta}^{\perp}\right)$, then $D_{\varphi}^{\theta} D_{z}^{\theta}-D_{z}^{\theta} D_{\varphi}^{\theta}=\theta \otimes C_{\theta}\left(P_{\theta}^{\perp}\left(\varphi k_{0}^{\theta}\right)\right)-\left(P_{\theta}^{\perp}\left(\varphi k_{0}^{\theta}\right)\right) \otimes \bar{z}$;

(2) if $A_{\varphi}^{\theta} \in \mathscr{B}\left(K_{\theta}\right)$, then $A_{\varphi}^{\theta} A_{z}^{\theta}-A_{z}^{\theta} A_{\varphi}^{\theta}=k_{0}^{\theta} \otimes\left(C_{\theta} P_{\theta}(\theta \varphi)\right)-\left(P_{\theta}(\theta \varphi)\right) \otimes C_{\theta} k_{0}^{\theta}$; 
(3) if $B_{\varphi}^{\theta} \in \mathscr{B}\left(K_{\theta}, K_{\theta}^{\perp}\right)$, then $B_{\varphi}^{\theta} A_{z}^{\theta}-D_{z}^{\theta} B_{\varphi}^{\theta}=\theta \otimes\left(C_{\theta} P_{\theta}\left(\varphi k_{0}^{\theta}\right)\right)-\left(P_{\theta}^{\perp}(\varphi \theta)\right) \otimes$ $C_{\theta} k_{0}^{\theta}$.

Proof. Note that if $f \in D\left(M_{\varphi}\right)$, then also $z f \in D\left(M_{\varphi}\right)$ and as a consequence we get commutativity $M_{z} M_{\varphi}=M_{\varphi} M_{z}$ on $D\left(M_{\varphi}\right)$. Observe that by Corollary $7, B_{z}^{\theta}\left(K_{\theta}^{\infty}\right) \subset \theta H^{\infty} \oplus \bar{z} \overline{H^{\infty}}$ and $\left(B_{\bar{z}}^{\theta}\right)^{*}\left(\theta H^{\infty} \oplus \bar{z} \overline{H^{\infty}}\right) \subset K_{\theta}^{\infty}$. Moreover, for $f, g \in H^{\infty}$ and $h \in K_{\theta}^{\infty}$ we have

$$
D_{z}^{\theta}(\theta f+\bar{z} \bar{g})=z \theta f+\overline{g-g(0)}+\overline{g(0)} \theta \overline{\theta(0)} \in \theta H^{\infty} \oplus \bar{z} \overline{H^{\infty}}
$$

(see Example 9) and

$$
A_{z}^{\theta} h=P_{\theta}(z h)=z h-\theta P^{+}(\bar{\theta} z h)=z h-\theta \cdot \overline{\left(C_{\theta} h\right)(0)} \in K_{\theta}^{\infty},
$$

that is, $D_{z}^{\theta}\left(\theta H^{\infty} \oplus \bar{z} \overline{H^{\infty}}\right) \subset \theta H^{\infty} \oplus \bar{z} \overline{H^{\infty}}$ and $A_{z}^{\theta}\left(K_{\theta}^{\infty}\right) \subset K_{\theta}^{\infty}$. Therefore, the equality $M_{\varphi} M_{z}=M_{z} M_{\varphi}$ can be expressed on $K_{\theta}^{\infty} \oplus\left(\theta H^{\infty} \oplus \bar{z} \overline{H^{\infty}}\right)$ as

$$
\left[\begin{array}{cc}
A_{\varphi}^{\theta} & \left(B_{\bar{\varphi}}^{\theta}\right)^{*} \\
B_{\varphi}^{\theta} & D_{\varphi}^{\theta}
\end{array}\right] \cdot\left[\begin{array}{cc}
A_{z}^{\theta} & \left(B_{\bar{z}}^{\theta}\right)^{*} \\
B_{z}^{\theta} & D_{z}^{\theta}
\end{array}\right]=\left[\begin{array}{cc}
A_{z}^{\theta} & \left(B_{\bar{z}}^{\theta}\right)^{*} \\
B_{z}^{\theta} & D_{z}^{\theta}
\end{array}\right] \cdot\left[\begin{array}{cc}
A_{\varphi}^{\theta} & \left(B_{\bar{\varphi}}^{\theta}\right)^{*} \\
B_{\varphi}^{\theta} & D_{\varphi}^{\theta}
\end{array}\right]
$$

Hence

$$
\begin{aligned}
B_{\varphi}^{\theta}\left(B_{\bar{z}}^{\theta}\right)^{*}+D_{\varphi}^{\theta} D_{z}^{\theta} & =B_{z}^{\theta}\left(B_{\bar{\varphi}}^{\theta}\right)^{*}+D_{z}^{\theta} D_{\varphi}^{\theta} \quad \text { on } \theta H^{\infty} \oplus \bar{z} \overline{H^{\infty}}, \\
A_{\varphi}^{\theta} A_{z}^{\theta}+\left(B_{\bar{\varphi}}^{\theta}\right)^{*} B_{z}^{\theta} & =A_{z}^{\theta} A_{\varphi}^{\theta}+\left(B_{\bar{z}}^{\theta}\right)^{*} B_{\varphi}^{\theta} \quad \text { on } K_{\theta}^{\infty}, \\
B_{\varphi}^{\theta} A_{z}^{\theta}+D_{\varphi}^{\theta} B_{z}^{\theta} & =B_{z}^{\theta} A_{\varphi}^{\theta}+D_{z}^{\theta} B_{\varphi}^{\theta} \quad \text { on } K_{\theta}^{\infty} .
\end{aligned}
$$

By Corollary 7 and Lemma 10, it follows from (4.3) that on $\theta H^{\infty} \oplus \bar{z} \overline{H^{\infty}}$,

$$
\begin{aligned}
D_{\varphi}^{\theta} D_{z}^{\theta}-D_{z}^{\theta} D_{\varphi}^{\theta} & =B_{z}^{\theta}\left(B_{\bar{\varphi}}^{\theta}\right)^{*}-B_{\varphi}^{\theta}\left(B_{\bar{z}}^{\theta}\right)^{*} \\
& =\left(\theta \otimes \tilde{k}_{0}^{\theta}\right)\left(B_{\bar{\varphi}}^{\theta}\right)^{*}-B_{\varphi}^{\theta}\left(k_{0}^{\theta} \otimes \bar{z}\right)=\theta \otimes\left(B_{\bar{\varphi}}^{\theta} \tilde{k}_{0}^{\theta}\right)-\left(B_{\varphi}^{\theta} k_{0}^{\theta}\right) \otimes \bar{z} \\
& =\theta \otimes C_{\theta}\left(P_{\theta}^{\perp}\left(\varphi k_{0}^{\theta}\right)\right)-\left(P_{\theta}^{\perp}\left(\varphi k_{0}^{\theta}\right)\right) \otimes \bar{z} .
\end{aligned}
$$

Hence (1) holds.

Similarly, from (4.4) it follows that on $K_{\theta}^{\infty}$,

$$
\begin{aligned}
A_{\varphi}^{\theta} A_{z}^{\theta}-A_{z}^{\theta} A_{\varphi}^{\theta}= & \left(B_{\bar{z}}^{\theta}\right)^{*} B_{\varphi}^{\theta}-\left(B_{\bar{\varphi}}^{\theta}\right)^{*} B_{z}^{\theta} \\
= & \left(k_{0}^{\theta} \otimes \bar{z}\right) B_{\varphi}^{\theta}-\left(B_{\bar{\varphi}}^{\theta}\right)^{*}\left(\theta \otimes \tilde{k}_{0}^{\theta}\right)=k_{0}^{\theta} \otimes\left(\left(B_{\varphi}^{\theta}\right)^{*} \bar{z}\right) \\
& -\left(\left(B_{\bar{\varphi}}^{\theta}\right)^{*} \theta\right) \otimes \tilde{k}_{0}^{\theta} \\
= & k_{0}^{\theta} \otimes\left(C_{\theta} P_{\theta}(\theta \varphi)\right)-\left(P_{\theta}(\theta \varphi)\right) \otimes C_{\theta} k_{0}^{\theta}
\end{aligned}
$$

and (2) is satisfied.

Finally, from (4.5) we conclude that on $K_{\theta}^{\infty}$,

$$
\begin{aligned}
B_{\varphi}^{\theta} A_{z}^{\theta}-D_{z}^{\theta} B_{\varphi}^{\theta}= & B_{z}^{\theta} A_{\varphi}^{\theta}-D_{\varphi}^{\theta} B_{z}^{\theta}=\left(\theta \otimes \tilde{k}_{0}^{\theta}\right) A_{\varphi}^{\theta}-D_{\varphi}^{\theta}\left(\theta \otimes \tilde{k}_{0}^{\theta}\right) \\
= & \theta \otimes\left(A_{\bar{\varphi}}^{\theta} C_{\theta} k_{0}^{\theta}\right)-\left(D_{\varphi}^{\theta} \theta\right) \otimes \tilde{k}_{0}^{\theta}=\theta \otimes\left(C_{\theta} A_{\varphi}^{\theta} k_{0}^{\theta}\right) \\
& -\left(P_{\theta}^{\perp}(\varphi \theta)\right) \otimes \tilde{k}_{0}^{\theta} \\
= & \theta \otimes\left(C_{\theta} P_{\theta}\left(\varphi k_{0}^{\theta}\right)\right)-\left(P_{\theta}^{\perp}(\varphi \theta)\right) \otimes C_{\theta} k_{0}^{\theta}
\end{aligned}
$$


and (3) follows.

As a corollary we get a necessary and sufficient condition for a dual truncated Toeplitz operator to commute with $D_{z}^{\theta}$.

Corollary 12. Let $D_{\varphi}^{\theta} \in \mathscr{T}\left(K_{\theta}^{\perp}\right)$. Then $D_{\varphi}^{\theta}$ commutes with $D_{z}^{\theta}$, i.e., $D_{\varphi}^{\theta} D_{z}^{\theta}=$ $D_{z}^{\theta} D_{\varphi}^{\theta}$ if and only if $\varphi k_{0}^{\theta}=c \theta+P_{\theta} \varphi$ for $c \in \mathbb{C}$. In particular, if $\theta(0)=0$, then $D_{\varphi}^{\theta} D_{z}^{\theta}=D_{z}^{\theta} D_{\varphi}^{\theta}$ if and only if $\varphi \in K_{\theta}+\mathbb{C} \theta$.

Proof. By Theorem 11,

$$
D_{\varphi}^{\theta} D_{z}^{\theta}=D_{z}^{\theta} D_{\varphi}^{\theta}
$$

if and only if

$$
\theta \otimes C_{\theta}\left(P_{\theta}^{\perp}\left(\varphi k_{0}^{\theta}\right)\right)=\left(P_{\theta}^{\perp}\left(\varphi k_{0}^{\theta}\right)\right) \otimes \bar{z}=\left(P_{\theta}^{\perp}\left(\varphi k_{0}^{\theta}\right)\right) \otimes C_{\theta} \theta .
$$

The above holds if and only if there is $c \in \mathbb{C}$ such that $P_{\theta}^{\perp}\left(\varphi k_{0}^{\theta}\right)=c \theta$, which is equivalent to $C_{\theta}\left(P_{\theta}^{\perp}\left(\varphi k_{0}^{\theta}\right)\right)=\bar{c} \bar{z}$. In other words, it holds if and only if $\varphi k_{0}^{\theta}=c \theta+g$ for some $g \in K_{\theta}$. It follows that $\varphi(1-\overline{\theta(0)} \theta)=\psi \in H^{2}$. Since $(1-\overline{\theta(0)} \theta)^{-1}$ is a bounded analytic function, we also have $\varphi=(1-\overline{\theta(0)} \theta)^{-1} \psi \in$ $H^{2}$. Hence

$$
P_{\theta}(\varphi)=P_{\theta}\left(\varphi k_{0}^{\theta}+\overline{\theta(0)} \theta \varphi\right)=P_{\theta}(c \theta+g+\overline{\theta(0)} \theta \varphi)=g,
$$

that is, $\varphi k_{0}^{\theta}=c \theta+P_{\theta}(\varphi)$.

Remark 13. The known fact that a truncated Toeplitz operator which commutes with $A_{z}^{\theta}$ has an analytic symbol (see [23]) can also be obtained from Theorem 11. Recall that $A_{\varphi}^{\theta}=0$ if and only if $\varphi \in \theta H^{2}+\overline{\theta H^{2}}$ ([22, Theorem 3.1]). Hence we can assume that $A_{\varphi}^{\theta}=A_{\bar{z} \bar{\chi}+\psi}^{\theta}$ with $\chi, \psi \in K_{\theta}$. From Theorem 11 it follows that $A_{\varphi}^{\theta} A_{z}^{\theta}=A_{z}^{\theta} A_{\varphi}^{\theta}$ if and only if for some $c \in \mathbb{C}$ we have

$$
c \tilde{k}_{0}^{\theta}=c C_{\theta} k_{0}^{\theta}=\left(B_{\varphi}^{\theta}\right)^{*} \bar{z}=\chi
$$

by Lemma 10 . Now, since $A_{\bar{\theta}}^{\theta}=0$, we have

$$
A_{\varphi}^{\theta}=A_{\bar{z} \bar{\chi}+\psi}^{\theta}=A_{\bar{c}(\bar{\theta}-\overline{\theta(0)})+\psi}^{\theta}=A_{\psi-\bar{c} \overline{\theta(0)}}^{\theta}
$$

and $A_{\varphi}^{\theta}$ has an analytic symbol $\psi-\bar{c} \overline{\theta(0)}$.

Next we get a necessary and sufficient condition for an operator in $\mathscr{T}\left(K_{\theta}, K_{\theta}^{\perp}\right)$ to intertwine $D_{z}^{\theta}$ and $A_{z}^{\theta}$.

Corollary 14. Let $B_{\varphi}^{\theta} \in \mathscr{B}\left(K_{\theta}, K_{\theta}^{\perp}\right)$. The operator $B_{\varphi}^{\theta}$ intertwines $A_{z}^{\theta}$ and $D_{z}^{\theta}$, i.e., $B_{\varphi}^{\theta} A_{z}^{\theta}=D_{z}^{\theta} B_{\varphi}^{\theta}$ if and only if

(1) $\varphi=c$ for some $c \in \mathbb{C}$, if $\theta(0) \neq 0$;

(2) $\varphi \in \bar{\theta} K_{\theta}+\mathbb{C}$, if $\theta(0)=0$.

Note that in the first case $B_{\varphi}^{\theta}=0$ and in the second $B_{\varphi}^{\theta}=B_{\varphi_{1}}^{\theta}$ with $\varphi_{1} \in \bar{\theta} K_{\theta}$. 
Proof. Theorem 11 (3) says that $B_{\varphi}^{\theta} A_{z}^{\theta}=D_{z}^{\theta} B_{\varphi}^{\theta}$ if and only if

$$
\theta \otimes\left(C_{\theta} P_{\theta}\left(\varphi k_{0}^{\theta}\right)\right)=\left(P_{\theta}^{\perp}(\varphi \theta)\right) \otimes C_{\theta} k_{0}^{\theta} .
$$

There are two possible cases

(i) $P_{\theta}\left(\varphi k_{0}^{\theta}\right)=0$ and $P_{\theta}^{\perp}(\varphi \theta)=0$, or

(ii) $\left.C_{\theta} P_{\theta}\left(\varphi k_{0}^{\theta}\right)\right)=\bar{c} C_{\theta} k_{0}^{\theta}$ and $P_{\theta}^{\perp}(\varphi \theta)=c \theta$ for some $c \in \mathbb{C}$.

Consider the first case. Then $\varphi k_{0}^{\theta}=\varphi-\overline{\theta(0)} \varphi \theta \perp K_{\theta}$ and $\varphi \theta \perp \theta H^{2}$. Note that by the latter, $\varphi \perp H^{2} \supset K_{\theta}$. Thus, if $\theta(0) \neq 0$, then $\varphi \theta \perp K_{\theta}$ which, together with $P_{\theta}^{\perp}(\varphi \theta)=0$, implies that $\varphi \theta=0$, i.e., $\varphi=0$. If $\theta(0)=0$, then $P_{\theta}^{\perp}(\varphi \theta)=0$ implies $\varphi \theta \in K_{\theta}$, i.e., $\varphi \in \bar{\theta} K_{\theta}$.

If (ii) is fulfilled, then

$$
P_{\theta}\left((\varphi-c) k_{0}^{\theta}\right)=0 \quad \text { and } \quad P_{\theta}^{\perp}((\varphi-c) \theta)=0,
$$

which, reasoning as previously, gives (1) or (2).

\section{Other Relations with Compressions of $M_{z}$}

If we consider the whole space $L^{2}$ and an operator $A \in \mathscr{B}\left(L^{2}\right)$, then $A$ fulfils the relation $A=M_{z}^{*} A M_{z}$ if and only if $A$ commutes with $M_{z}$. On the other hand, it is not the case when we consider the space $H^{2}$ and the unilateral shift $T_{z}$ as a compression of $M_{z}$. The classical Brown-Halmos result shows that a bounded linear operator $T \in \mathscr{B}\left(H^{2}\right)$ is a Toeplitz operator if and only if $T=\left(T_{z}\right)^{*} T T_{z}$. Similar characterizations (in terms of compressions of $M_{z}$ ) are known for Hankel operators and dual Toeplitz operators. In [22] D. Sarason characterized bounded truncated Toeplitz operators in terms of the compressions of $M_{z}$ to $K_{\theta}$. In particular, he proved that a bounded operator $A \in \mathscr{B}\left(K_{\theta}\right)$ is a truncated Toeplitz operator if and only if

$$
A-A_{z}^{\theta} A A_{\bar{z}}^{\theta}=\psi \otimes k_{0}^{\theta}+k_{0}^{\theta} \otimes \chi
$$

for some $\psi, \chi \in K_{\theta}$. In other words, the left hand side of (5.1) can be expressed as an operator of rank at most two. In this section our aim is to give similar expressions for operators from $\mathscr{T}\left(K_{\theta}, K_{\theta}^{\perp}\right)$ and $\mathscr{T}\left(K_{\theta}^{\perp}\right)$ using operators of rank at most two.

Proposition 15. Let $D_{\varphi}^{\theta} \in \mathscr{T}\left(K_{\theta}^{\perp}\right)$. Then

$$
D_{\varphi}^{\theta}-D_{z}^{\theta} D_{\varphi}^{\theta} D_{\bar{z}}^{\theta}=C_{\theta} P_{\theta}^{\perp}\left(\bar{\varphi} \bar{z} k_{0}^{\theta}\right) \otimes \theta+\theta \otimes C_{\theta} P_{\theta}^{\perp}\left(\bar{z} P_{\theta}^{\perp}\left(\varphi k_{0}^{\theta}\right)\right) .
$$

Remark 16. The characterization (5.1) proved in [22] for truncated Toeplitz operators immediately gives a symbol of the truncated Toeplitz operator $A=$ $A_{\psi+\bar{\chi}}^{\theta}$. Moreover, the relation between $\psi$ and $\chi$ is simple, see [22, Corollary after Theorem 3.1]. However, for any dual truncated Toeplitz operator, the functions $\mu=C_{\theta} P_{\theta}^{\perp}\left(\bar{\varphi} \bar{z} k_{0}^{\theta}\right), \nu=C_{\theta} P_{\theta}^{\perp}\left(\bar{z} P_{\theta}^{\perp}\left(\varphi k_{0}^{\theta}\right)\right) \in K_{\theta}^{\perp}$ in the formula (5.2), strongly and in a very complicated way depend on each other. Moreover, in case of dual truncated Toeplitz operators, having the rank-two operator on 
the right hand side of (5.2), $\mu \otimes \theta+\theta \otimes \nu$ with $\mu, \nu \in K_{\theta}^{\perp}$ we are far from obtaining the symbol of $D$. For this reason, to answer a natural question when an operator $D \in \mathscr{B}\left(K_{\theta}^{\perp}\right)$ is a DTTO and to find its symbol, we will consider restrictions of $D$ to some subspaces of $K_{\theta}^{\perp}$. This will be done in Theorem 27 .

Proof of Proposition 15. By Theorem 11 (1) we have since

$$
D_{\varphi}^{\theta} D_{z}^{\theta} D_{\bar{z}}^{\theta}-D_{z}^{\theta} D_{\varphi}^{\theta} D_{\bar{z}}^{\theta}=\theta \otimes D_{z}^{\theta} C_{\theta} P_{\theta}^{\perp}\left(\varphi k_{0}^{\theta}\right)-P_{\theta}^{\perp}\left(\varphi k_{0}^{\theta}\right) \otimes D_{z}^{\theta} \bar{z} .
$$

$D_{z}^{\theta} \bar{z}=P_{\theta}^{\perp} 1=\overline{\theta(0)} \theta$, using Proposition 8 (1) we get

$$
\begin{aligned}
& D_{\varphi}^{\theta}-D_{z}^{\theta} D_{\varphi}^{\theta} D_{\bar{z}}^{\theta} \\
& \quad=\left(\left(1-|\theta(0)|^{2}\right) D_{\varphi}^{\theta} \theta-\theta(0) P_{\theta}^{\perp}\left(\varphi k_{0}^{\theta}\right)\right) \otimes \theta+\theta \otimes D_{z}^{\theta} C_{\theta} P_{\theta}^{\perp}\left(\varphi k_{0}^{\theta}\right) .
\end{aligned}
$$

Since $D_{\varphi}^{\theta}(\theta)=P_{\theta}^{\perp}(\theta \varphi)$, we obtain

$$
\begin{aligned}
\left(1-|\theta(0)|^{2}\right) D_{\varphi}^{\theta} \theta- & \theta(0) P_{\theta}^{\perp}\left(\varphi k_{0}^{\theta}\right)=P_{\theta}^{\perp}\left(\theta \varphi-|\theta(0)|^{2} \theta \varphi-\theta(0) \varphi k_{0}^{\theta}\right) \\
& =P_{\theta}^{\perp}\left(\theta \varphi-|\theta(0)|^{2} \theta \varphi-\theta(0) \varphi+|\theta(0)|^{2} \theta \varphi\right) \\
& =P_{\theta}^{\perp}(\varphi(\theta-\theta(0)))=P_{\theta}^{\perp} C_{\theta}\left(\bar{\varphi} \bar{z} k_{0}^{\theta}\right)=C_{\theta} P_{\theta}^{\perp}\left(\bar{\varphi} \bar{z} k_{0}^{\theta}\right) .
\end{aligned}
$$

The proof will be completed with

$$
D_{z}^{\theta} C_{\theta} P_{\theta}^{\perp}\left(\varphi k_{0}^{\theta}\right)=P_{\theta}^{\perp}\left(z C_{\theta} P_{\theta}^{\perp}\left(\varphi k_{0}^{\theta}\right)\right)=C_{\theta} P_{\theta}^{\perp}\left(\bar{z} P_{\theta}^{\perp}\left(\varphi k_{0}^{\theta}\right)\right) .
$$

Proposition 17. Let $B_{\varphi}^{\theta} \in \mathscr{T}\left(K_{\theta}, K_{\theta}^{\perp}\right)$. Then

$$
B_{\varphi}^{\theta}-D_{z}^{\theta} B_{\varphi}^{\theta} A_{\bar{z}}^{\theta}=\theta \otimes\left(P_{\theta}\left(z P_{\theta}\left(\bar{\varphi} \tilde{k}_{0}^{\theta}\right)\right)\right)+\left(P_{\theta}^{\perp} \varphi\right) \otimes k_{0}^{\theta} .
$$

Proof. By Theorem 11 (3) we have

$$
B_{\varphi}^{\theta} A_{z}^{\theta} A_{\bar{z}}^{\theta}-D_{z}^{\theta} B_{\varphi}^{\theta} A_{\bar{z}}^{\theta}=\theta \otimes\left(A_{z}^{\theta} C_{\theta} P_{\theta}\left(\varphi k_{0}^{\theta}\right)\right)-\left(P_{\theta}^{\perp}(\varphi \theta)\right) \otimes A_{z}^{\theta} C_{\theta} k_{0}^{\theta} .
$$

By (3.2) it follows that

$$
B_{\varphi}^{\theta}\left(I_{K_{\theta}}-k_{0}^{\theta} \otimes k_{0}^{\theta}\right)-D_{z}^{\theta} B_{\varphi}^{\theta} A_{\bar{z}}^{\theta}=\theta \otimes\left(A_{z}^{\theta} P_{\theta}\left(\bar{\varphi} \widetilde{k}_{0}^{\theta}\right)\right)-\left(P_{\theta}^{\perp}(\varphi \theta)\right) \otimes\left(A_{z}^{\theta} \tilde{k}_{0}^{\theta}\right) .
$$

Since $A_{z}^{\theta} \tilde{k}_{0}^{\theta}=-\theta(0) k_{0}^{\theta}$ (see $\left[22\right.$, Lemma 2.2]) and $A_{z}^{\theta} P_{\theta}\left(\bar{\varphi} \widetilde{k}_{0}^{\theta}\right)=P_{\theta}\left(z P_{\theta}\left(\bar{\varphi} \tilde{k}_{0}^{\theta}\right)\right)$, we have finally,

$$
\begin{gathered}
B_{\varphi}^{\theta}-D_{z}^{\theta} B_{\varphi}^{\theta} A_{\bar{z}}^{\theta}=\theta \otimes\left(P_{\theta}\left(z P_{\theta}\left(\bar{\varphi} \tilde{k}_{0}^{\theta}\right)\right)\right)+\overline{\theta(0)}\left(P_{\theta}^{\perp}(\varphi \theta)\right) \otimes k_{0}^{\theta}+\left(B_{\varphi}^{\theta} k_{0}^{\theta}\right) \otimes k_{0}^{\theta} . \\
B_{\varphi}^{\theta} k_{0}^{\theta}+\overline{\theta(0)} P_{\theta}^{\perp}(\varphi \theta)=P_{\theta}^{\perp}(\varphi(1-\overline{\theta(0)} \theta))+\overline{\theta(0)} P_{\theta}^{\perp}(\varphi \theta)=P_{\theta}^{\perp} \varphi
\end{gathered}
$$

which completes the proof.

Corollary 18. For each $\varphi \in L^{2}$ :

1. if $D_{\varphi}^{\theta} \in \mathscr{T}\left(K_{\theta}^{\perp}\right)$, then $D_{\varphi}^{\theta}-D_{\bar{z}}^{\theta} D_{\varphi}^{\theta} D_{z}^{\theta}=P_{\theta}^{\perp}\left(\varphi \bar{z} k_{0}^{\theta}\right) \otimes \bar{z}+\bar{z} \otimes P_{\theta}^{\perp}\left(\bar{z} P_{\theta}^{\perp}\left(\bar{\varphi} k_{0}^{\theta}\right)\right)$;

2. if $B_{\varphi}^{\theta} \in \mathscr{T}\left(K_{\theta}, K_{\theta}^{\perp}\right)$, then $B_{\varphi}^{\theta}-D_{\bar{z}}^{\theta} B_{\varphi}^{\theta} A_{z}^{\theta}=C_{\theta} P_{\theta}^{\perp} \bar{\varphi} \otimes \widetilde{k}_{0}^{\theta}+\bar{z} \otimes P_{\theta}\left(\bar{z} P_{\theta}\left(\bar{\varphi} k_{0}^{\theta}\right)\right)$. 
Proof. We apply the conjugation $C_{\theta}$ on (5.2) and (5.4) substituting $\varphi$ with $\bar{\varphi}$. Then by Corollary 2, we have

$$
D_{\varphi}^{\theta}-D_{\bar{z}}^{\theta} D_{\varphi}^{\theta} D_{z}^{\theta}=P_{\theta}^{\perp}\left(\varphi \bar{z} k_{0}^{\theta}\right) \otimes C_{\theta} \theta+C_{\theta} \theta \otimes P_{\theta}^{\perp}\left(\bar{z} P_{\theta}^{\perp}\left(\bar{\varphi} k_{0}^{\theta}\right)\right) .
$$

Now (1) follows, since $C_{\theta} \theta=\bar{z}$. Similarly,

$$
B_{\varphi}^{\theta}-D_{\bar{z}}^{\theta} B_{\varphi}^{\theta} A_{z}^{\theta}=\bar{z} \otimes C_{\theta}\left(P_{\theta}\left(z P_{\theta}\left(\bar{\varphi} \tilde{k}_{0}^{\theta}\right)\right)\right)+C_{\theta} P_{\theta}^{\perp} \bar{\varphi} \otimes \widetilde{k}_{0}^{\theta} .
$$

We obtain (2) showing that

$$
\begin{aligned}
C_{\theta}\left(P_{\theta}\left(z P_{\theta}\left(\varphi \tilde{k}_{0}^{\theta}\right)\right)\right) & =P_{\theta} C_{\theta}\left(z P_{\theta} C_{\theta}\left(\bar{\varphi} k_{0}^{\theta}\right)\right) \\
& =P_{\theta} C_{\theta}\left(z C_{\theta} P_{\theta}\left(\bar{\varphi} k_{0}^{\theta}\right)\right)=P_{\theta}\left(\bar{z} P_{\theta}\left(\bar{\varphi} k_{0}^{\theta}\right)\right) .
\end{aligned}
$$

\section{Restrictions of $D_{\varphi}^{\theta}$}

Let $\theta$ be an inner function, and let $D \in \mathscr{B}\left(K_{\theta}^{\perp}\right)$. Using the decomposition $K_{\theta}^{\perp}=\theta H^{2} \oplus H_{-}^{2}$ we can write $D$ as a matrix

$$
D=\left[\begin{array}{cc}
P_{\theta H^{2}} D_{\mid \theta H^{2}} & P_{\theta H^{2}} D_{\mid H_{-}^{2}} \\
P^{-} D_{\mid \theta H^{2}} & P^{-} D_{\mid H_{-}^{2}}
\end{array}\right] .
$$

In particular, for $\varphi \in L^{\infty}$, we have

$$
D_{\varphi}^{\theta}=\left[\begin{array}{cc}
\hat{T}_{\varphi}^{\theta} & \check{\Gamma}_{\varphi}^{\theta} \\
\hat{\Gamma}_{\varphi}^{\theta} & \check{T}_{\varphi}
\end{array}\right]=\left[\begin{array}{cc}
\hat{T}_{\varphi}^{\theta} & \left(\hat{\Gamma}_{\bar{\varphi}}^{\theta}\right)^{*} \\
\hat{\Gamma}_{\varphi}^{\theta} & \check{T}_{\varphi}
\end{array}\right],
$$

where

$$
\begin{array}{ll}
\hat{T}_{\varphi}^{\theta}=P_{\theta H^{2}} M_{\varphi \mid \theta H^{2}}, & \check{\Gamma}_{\varphi}^{\theta}=P_{\theta H^{2}} M_{\varphi \mid H_{-}^{2}}, \\
\hat{\Gamma}_{\varphi}^{\theta}=P^{-} M_{\varphi \mid \theta H^{2}}, & \check{T}_{\varphi}=P^{-} M_{\varphi \mid H_{-}^{2}} .
\end{array}
$$

This notation will be used in the following sections. We also set the notation

$$
\begin{aligned}
\mathscr{T}\left(\theta H^{2}\right) & =\left\{\hat{T} \in \mathscr{B}\left(\theta H^{2}\right): \hat{T}=\hat{T}_{\varphi}^{\theta} \text { for some } \varphi \in L^{\infty}\right\}, \\
\mathscr{T}\left(H_{-}^{2}\right) & =\left\{\check{T} \in \mathscr{B}\left(H_{-}^{2}\right): \check{T}=\check{T}_{\varphi} \text { for some } \varphi \in L^{\infty}\right\}, \\
\mathscr{T}\left(\theta H^{2}, H_{-}^{2}\right) & =\left\{\hat{\Gamma} \in \mathscr{B}\left(\theta H^{2}, H_{-}^{2}\right): \hat{\Gamma}=\hat{\Gamma}_{\varphi}^{\theta}=P^{-} M_{\varphi \mid \theta H^{2}} \text { for } \varphi \in L^{\infty}\right\}, \\
\mathscr{T}\left(H_{-}^{2}, \theta H^{2}\right) & =\left\{\check{\Gamma} \in \mathscr{B}\left(H_{-}^{2}, \theta H^{2}\right): \check{\Gamma}=\check{\Gamma}_{\varphi}^{\theta}=P_{\theta H^{2}} M_{\varphi \mid H_{-}^{2}} \text { for } \varphi \in L^{\infty}\right\} .
\end{aligned}
$$

Note that $\check{\Gamma}_{\varphi}^{\theta}=\left(\hat{\Gamma}_{\bar{\varphi}}^{\theta}\right)^{*}$ and so $\check{\Gamma} \in \mathscr{T}\left(H_{-}^{2}, \theta H^{2}\right)$ if and only if $\check{\Gamma}^{*} \in \mathscr{T}\left(\theta H^{2}, H_{-}^{2}\right)$.

It is a part of common knowledge that the space of classical Toeplitz operators $\mathscr{T}\left(H^{2}\right)$ is isomorphic to the space $\mathscr{T}\left(\theta H^{2}\right)$ and to $\mathscr{T}\left(H_{-}^{2}\right)$, but we present some lemmas for completeness and to fix the notations of these spacial isomorphisms. Recall also the notation $\mathscr{H}\left(H^{2}, H_{-}^{2}\right)$ for the space of all Hankel operators.

Consider the operator $J$, where $J: L^{2} \rightarrow L^{2}, J f(z)=\bar{z} \overline{f(z)}, z \in \mathbb{T}$. Note that $J$ maps antilinearly $H^{2}$ onto $H_{-}^{2}$ and conversely bijectively. Moreover, 
$J^{-1}=J=J^{\sharp}$ (by $\sharp$ we denote the antilinear adjoint). For more properties of antilinear operators authors send the reader to [19]. Consider also the operator $M_{\theta}$ and note that $M_{\theta}$ maps $H^{2}$ onto $\theta H^{2}$ bijectively. Moreover $M_{\theta}^{-1}=M_{\bar{\theta}}$.

The following properties can be easily verified.

Proposition 19. (1) $\left\langle f_{1}, f_{2}\right\rangle=\left\langle\theta f_{1}, \theta f_{2}\right\rangle=\left\langle J f_{2}, J f_{1}\right\rangle$ for $f_{1}, f_{2} \in L^{2}$;

(2) $P_{\theta H^{2}}=M_{\theta} P^{+} M_{\bar{\theta}}$;

(3) $P^{-}=J P^{+} J$;

(4) $M_{\theta}\left(f_{1} \otimes f_{2}\right) M_{\bar{\theta}}=\theta f_{1} \otimes \theta f_{2}$ for $f_{1}, f_{2} \in L^{2}$;

(5) $J\left(f_{1} \otimes f_{2}\right) J=J f_{1} \otimes J f_{2}$ for $f_{1}, f_{2} \in L^{2}$;

(6) $M_{\theta} J M_{\theta}=J$;

(7) $J M_{\varphi}=M_{\bar{\varphi}} J$ for $\varphi \in L^{\infty}$.

In particular $J 1=\bar{z}$ and $J(1 \otimes 1) J=\bar{z} \otimes \bar{z}$. From these properties we get:

Proposition 20. Let $\varphi \in L^{\infty}$. Then

(1) $\hat{T}_{\varphi}^{\theta}=P_{\theta H^{2}} M_{\varphi \mid \theta H^{2}}=M_{\theta} T_{\varphi} M_{\bar{\theta} \mid \theta H^{2}}$;

(2) $\hat{T}_{\varphi}^{\theta}=M_{\varphi \mid \theta H^{2}}=T_{\varphi \mid \theta H^{2}}$ if $\varphi \in H^{\infty}$;

(3) $\check{T}_{\varphi}=P^{-} M_{\varphi \mid H_{-}^{2}}=J T_{\bar{\varphi}} J_{\mid H_{-}^{2}}$;

(4) $\check{T}_{\varphi}=M_{\varphi \mid H_{-}^{2}}$, if $\varphi \in \overline{H^{\infty}}$;

(5) $\hat{\Gamma}_{\varphi}^{\theta}=P^{-} M_{\varphi \mid \theta H^{2}}=H_{\varphi \theta} M_{\bar{\theta} \mid \theta H^{2}}$;

(6) $\check{\Gamma}_{\varphi}^{\theta}=P_{\theta H^{2}} M_{\varphi \mid H_{-}^{2}}=M_{\theta} H_{\theta \bar{\varphi}}^{*}$.

This proposition shows that the symbols of $\hat{T}_{\varphi}^{\theta}$ and $\check{T}_{\varphi}$ are unique, and that $\hat{\Gamma}_{\varphi}^{\theta}$ and $\check{\Gamma}_{\varphi}^{\theta}$ are uniquely determined by $P^{-}(\varphi \theta)$ and $P^{-}(\theta \bar{\varphi})$, respectively. Note that for $\varphi \in L^{\infty}, \bar{\theta} P^{-}(\theta \varphi)$ is the orthogonal projection of $\varphi$ onto $\overline{\theta z H^{2}}$. Thus

$$
\hat{\Gamma}_{\varphi}=\hat{\Gamma}_{\psi} \quad \text { if and only if } \quad(\varphi-\psi) \perp \overline{\theta z H^{2}} .
$$

In particular, $\hat{\Gamma}_{\varphi}^{\theta}=0$ if $\varphi \in \bar{\theta} H^{\infty} \supset H^{\infty}$. Similarly, $\check{\Gamma}_{\varphi}^{\theta}=0$ if $\varphi \in \theta \overline{H^{\infty}} \supset \overline{H^{\infty}}$. Proposition 20 implies the following.

Corollary 21. (1) $\hat{T}_{z}^{\theta}=M_{z \mid \theta H^{2}}, \hat{T}_{\bar{z}}^{\theta}=M_{\bar{z} \mid \theta H^{2}}-(\theta \bar{z} \otimes \theta)_{\mid \theta H^{2}}$;

(2) $\check{T}_{z}=M_{z \mid H_{-}^{2}}-(1 \otimes \bar{z})_{\mid H_{-}^{2}}, \check{T}_{\bar{z}}=M_{\bar{z} \mid H_{-}^{2}}$;

(3) $\hat{\Gamma}_{z}^{\theta}=0, \hat{\Gamma}_{\bar{z}}^{\theta}=(\bar{z} \otimes 1)_{\mid \theta H^{2}}, \hat{\Gamma}_{\bar{z}}^{\theta}=0$ if $\theta(0)=0$;

(4) $\check{\Gamma}_{z}^{\theta}=(\overline{\theta(0)} \theta \otimes \bar{z})_{\mid H_{-}^{2}}, \check{\Gamma}_{\bar{z}}^{\theta}=0, \check{\Gamma}_{z}^{\theta}=0$ if $\theta(0)=0$.

Proof. For (1) take $f \in \theta H^{2}$. Then

$$
\begin{aligned}
\hat{T}_{\bar{z}}^{\theta} f & =\theta P^{+} \bar{\theta} \bar{z} f=\theta P^{+} \bar{z}(\bar{\theta} f)=\theta \bar{z}(\bar{\theta} f-(\bar{\theta} f)(0)) \\
& =\bar{z} f-\theta \bar{z}\langle\bar{\theta} f, 1\rangle=\bar{z} f-\theta \bar{z}\langle f, \theta\rangle=\bar{z} f-(\theta \bar{z} \otimes \theta) f .
\end{aligned}
$$

To obtain (2) take $f \in H_{-}^{2}$,

$$
\check{T}_{z} f=P^{-} z f=z f-P^{+}(z f)=z f-\langle f, \bar{z}\rangle=z f-(1 \otimes \bar{z}) f .
$$


Observe that (3) follows, since for $f \in H^{2}$, we have

$$
\hat{\Gamma}_{\bar{z}}^{\theta}(\theta f)=P^{-} \bar{z} \theta f=\theta(0) f(0) \bar{z}=\langle\theta f, 1\rangle \bar{z}=(\bar{z} \otimes 1)(\theta f) .
$$

To note (4) take $f \in H_{-}^{2}$,

$$
\begin{aligned}
\check{\Gamma}_{z}^{\theta} f & =\theta P^{+} \bar{\theta} z f=\theta P^{+} z(\bar{\theta} f)=\theta\langle\bar{\theta} f, \bar{z}\rangle=\theta\langle f, \theta \bar{z}\rangle \\
& =\left\langle f, P^{-}(\theta \bar{z})\right\rangle \theta=\langle f, \theta(0) \bar{z}\rangle \theta=\overline{\theta(0)}\langle f, \bar{z}\rangle \theta=\overline{\theta(0)}(\theta \otimes \bar{z}) f .
\end{aligned}
$$
hence

The conjugation $C_{\theta}$ (see $\left.(2.1)\right)$ can be expressed as $C_{\theta}=M_{\theta} J=J M_{\bar{\theta}}$,

Proposition 22. For $\varphi \in L^{\infty}$,

(1) $\hat{T}_{\varphi}^{\theta}=C_{\theta} \check{T}_{\bar{\varphi}} C_{\theta \mid \theta H^{2}}$;

(2) $\hat{\Gamma}_{\varphi}^{\theta}=C_{\theta} \check{\Gamma}_{\bar{\varphi}}^{\theta} C_{\theta \mid \theta H^{2}}$.

Proof. To prove (1), note that by Propositions 19 and 20 we have

$$
\begin{aligned}
\hat{T}_{\varphi}^{\theta} & =M_{\theta} T_{\varphi} M_{\bar{\theta} \mid \theta H^{2}}=M_{\theta}\left(J P^{-} J\right) M_{\varphi} J\left(J M_{\bar{\theta}}\right)_{\mid \theta H^{2}} \\
& =\left(M_{\theta} J\right) P^{-} M_{\bar{\varphi}} P^{-} J M_{\bar{\theta} \mid \theta H^{2}}=C_{\theta} \check{T}_{\bar{\varphi}} C_{\theta \mid \theta H^{2}} .
\end{aligned}
$$

The condition (2) can be also obtained from the propositions above, since

$$
\begin{aligned}
\hat{\Gamma}_{\varphi}^{\theta} & =P^{-} M_{\varphi \mid \theta H^{2}}=\left(J P^{+} J\right) M_{\varphi} M_{\theta} J\left(J M_{\bar{\theta}}\right)_{\mid \theta H^{2}}=J P^{+} M_{\bar{\theta}} M_{\bar{\varphi}} C_{\theta \mid \theta H^{2}} \\
& =M_{\theta} J\left(M_{\theta} P^{+} M_{\bar{\theta}}\right) M_{\bar{\varphi}} P^{-} C_{\theta \mid \theta H^{2}}=C_{\theta} P_{\theta H^{2}} M_{\bar{\varphi}} P^{-} C_{\theta \mid \theta H^{2}}=C_{\theta} \check{\Gamma}_{\bar{\varphi}}^{\theta} C_{\theta \mid \theta H^{2}} .
\end{aligned}
$$

From Proposition 20 (1), (2) we obtain, in particular, that

Corollary 23. For $\varphi_{1}, \varphi_{2} \in L^{\infty}$,

(1) $\hat{T}_{\varphi_{1}}^{\theta} \hat{T}_{\varphi_{2}}^{\theta}=M_{\theta} T_{\varphi_{1}} T_{\varphi_{2}} M_{\bar{\theta} \mid \theta H^{2}}$;

(2) $\check{T}_{\varphi_{1}} \check{T}_{\varphi_{2}}=J T_{\bar{\varphi}_{1}} T_{\bar{\varphi}_{2}} J$;

(3) $\hat{T}_{\bar{z}}^{\theta} \hat{T}_{z}^{\theta}=I_{\theta H^{2}}$;

(4) $\hat{T}_{z}^{\theta} \hat{T}_{\bar{z}}^{\theta}=M_{\theta}(I-1 \otimes 1) M_{\bar{\theta} \mid \theta H^{2}}=I_{\theta H^{2}}-\theta \otimes \theta_{\mid \theta H^{2}}$;

(5) $\check{T}_{\bar{z}} \check{T}_{z}=J(I-1 \otimes 1) J_{\mid H_{-}^{2}}=I_{H_{-}^{2}}-\bar{z} \otimes \bar{z}_{\mid H_{-}^{2}}$;

(6) $\check{T}_{z} \check{T}_{\bar{z}}=I_{H^{2}}$.

Proposition 24. Let $\theta$ be a nonconstant inner function. Then

(1) $\hat{T} \in \mathscr{T}\left(\theta H^{2}\right)$ if and only if $M_{\bar{\theta}} \hat{T} M_{\theta \mid H^{2}} \in \mathscr{T}\left(H^{2}\right)$;

(2) $\check{T} \in \mathscr{T}\left(H_{-}^{2}\right)$ if and only if $J \check{T} J_{\mid H^{2}} \in \mathscr{T}\left(H^{2}\right)$;

(3) $\hat{\Gamma} \in \mathscr{T}\left(\theta H^{2}, H_{-}^{2}\right)$ if and only if $\hat{\Gamma} M_{\theta \mid H^{2}} \in \mathscr{H}\left(H^{2}, H_{-}^{2}\right)$;

(4) $\check{\Gamma} \in \mathscr{T}\left(H_{-}^{2}, \theta H^{2}\right)$ if and only if $\left(M_{\bar{\theta}} \check{\Gamma}\right)^{*} \in \mathscr{H}\left(H^{2}, H_{-}^{2}\right)$.

Recall that 
(A) if $T \in \mathscr{B}\left(H^{2}\right)$, then $T \in \mathscr{T}\left(H^{2}\right)$ if and only if $T=T_{z}^{*} T T_{z}$ and in that case $T=T_{\varphi}$ with $\left.\varphi=T(1)+\overline{T^{*}(1)-\left\langle T^{*} 1,1\right.}\right\rangle$, (it is Brown-Halmos result, see [11, Theorem 4.16]);

(B) if $H \in \mathscr{B}\left(H^{2}, H_{-}^{2}\right)$, then $H \in \mathscr{H}\left(H^{2}, H_{-}^{2}\right)$ if and only if $P^{-} z H=H T_{z}$ and in that case $P^{-} \varphi=H(1)$, see [18, Theorem 1.8, Chapter 1].

Using these results and Proposition 24 we get the following characterizations.

Theorem 25. Let $\theta$ be a nonconstant inner function.

(a) Let $\hat{T} \in \mathscr{B}\left(\theta H^{2}\right)$. Then $\hat{T} \in \mathscr{T}\left(\theta H^{2}\right)$ if and only if $\hat{T}=\hat{T}_{\bar{z}}^{\theta} \hat{T} \hat{T}_{z}^{\theta}$ and in that case $\hat{T}=\hat{T}_{\varphi}^{\theta}$ with $\varphi=\bar{\theta} \hat{T}(\theta)+\theta \overline{\hat{T}^{*}(\theta)}-\overline{\left\langle\hat{T}^{*} \theta, \theta\right\rangle}$.

(b) Let $\check{T} \in \mathscr{B}\left(H_{-}^{2}\right)$. Then $\check{T} \in \mathscr{T}\left(H_{-}^{2}\right)$ if and only if $\check{T}=\check{T}_{z} \check{T}_{\bar{T}}$ and, in that case, $\check{T}=\check{T}_{\varphi}$ with $\varphi=z \check{T} \bar{z}+\bar{z} \check{T}^{*} \bar{z}-\langle\check{T} \bar{z}, \bar{z}\rangle$.

(c) Let $\hat{\Gamma} \in \mathscr{B}\left(\theta H^{2}, H_{-}^{2}\right)$. Then $\hat{\Gamma} \in \mathscr{T}\left(\theta H^{2}, H_{-}^{2}\right)$ if and only if $\check{T}_{z} \hat{\Gamma}=\hat{\Gamma} \hat{T}_{z}^{\theta}$ and in that case $\hat{\Gamma}=\hat{\Gamma}_{\varphi}^{\theta}$ with $P^{-}(\theta \varphi)=\hat{\Gamma} \theta$.

(d) Let $\check{\Gamma} \in \mathscr{B}\left(H_{-}^{2}, \theta H^{2}\right)$. Then $\check{\Gamma} \in \mathscr{T}\left(H_{-}^{2}, \theta H^{2}\right)$ if and only if $\check{\Gamma}_{\bar{T}}=\hat{T}_{\bar{z}}^{\theta} \check{\Gamma}$ and in that case $\check{\Gamma}=\check{\Gamma}_{\varphi}^{\theta}$ with $P^{-}(\theta \bar{\varphi})=\check{\Gamma}^{*} \theta$.

Proof. By Proposition 24 (1) and (A), if $\hat{T} \in \mathscr{T}\left(\theta H^{2}\right)$, then $\mathscr{T}\left(H^{2}\right) \ni M_{\bar{\theta}} \hat{T}$ $M_{\theta \mid H^{2}}=T_{z}^{*} M_{\bar{\theta}} \hat{T} M_{\theta} T_{z}$. Equivalently $\hat{T}=\left(M_{\theta} T_{\bar{z}} M_{\bar{\theta}}\right) \hat{T}\left(M_{\theta} T_{z} M_{\bar{\theta}}\right)_{\mid \theta H^{2}}$ $=\hat{T}_{\bar{z}}^{\theta} \hat{T} \hat{T}_{z}^{\theta}$.

The symbol of $\hat{T}$ is the same as the symbol of $T=M_{\bar{\theta}} \hat{T} M_{\theta}$, so

$$
\begin{aligned}
\varphi & \left.=\left(M_{\bar{\theta}} \hat{T} M_{\theta}\right)(1)+\overline{\left(M_{\bar{\theta}} \hat{T} M_{\theta}\right)^{*}(1)-\left\langle\left(M_{\bar{\theta}} \hat{T} M_{\theta}\right)^{*} 1,1\right.}\right\rangle \\
& =\bar{\theta} \hat{T} \theta+\overline{\bar{\theta} \hat{T}^{*} \theta-\left\langle\bar{\theta} \hat{T}^{*} \theta, 1\right\rangle}=\bar{\theta} \hat{T} \theta+\theta \overline{\hat{T}^{*} \theta}-\overline{\left\langle\hat{T}^{*} \theta, \theta\right\rangle .}
\end{aligned}
$$

To see (b) note that by Proposition 24 (2) and (A), if $\check{T} \in \mathscr{T}\left(H_{-}^{2}\right)$, then $\mathscr{T}\left(H^{2}\right) \ni J \check{T} J_{\mid H^{2}}=T_{z}^{*} J \hat{T} J T_{z}$. Equivalently $\check{T}=\left(J T_{\bar{z}} J\right) \check{T} J T_{z} J_{\mid H_{-}^{2}}=\check{T}_{z} \check{T}_{\overline{\mathrm{T}}} \check{\bar{z}}_{\text {. }}$ In that case its symbol is the conjugate of the symbol of $T=J \check{T} J \in \mathscr{T}\left(H^{2}\right)$, hence

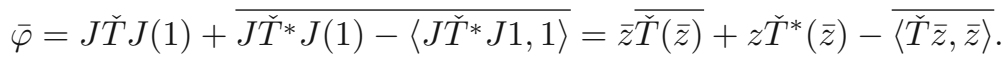

To prove (c) we apply Proposition 24 (3) and (B). We have that $\hat{\Gamma} \in$ $\mathscr{T}\left(\theta H^{2}, H_{-}^{2}\right)$ if and only if $P^{-} z \hat{\Gamma} M_{\theta \mid H^{2}}=\hat{\Gamma} M_{\theta} T_{z}$. Equivalently,

$$
\check{T}_{z} \hat{\Gamma}=P^{-} z P^{-} \hat{\Gamma}=\hat{\Gamma} M_{\theta} T_{z} M_{\bar{\theta} \mid \theta H^{2}}=\hat{\Gamma} \hat{T}_{z}^{\theta} .
$$

In that case $\hat{\Gamma}=\hat{\Gamma}_{\varphi}$ where $\theta \varphi$ is a symbol for the Hankel operator $\hat{\Gamma} M_{\theta \mid H^{2}}$ (by Proposition $20(5)$ ), thus $P^{-}(\theta \varphi)=\hat{\Gamma} M_{\theta}(1)=\hat{\Gamma} \theta$.

To obtain the last condition we apply Proposition 24 (4) and (B). Note that $\check{\Gamma} \in \mathscr{T}\left(H_{-}^{2}, \theta H^{2}\right)$ if and only if $\check{\Gamma}^{*} M_{\theta \mid H^{2}} \in \mathscr{H}\left(H^{2}, H_{-}^{2}\right)$. Equivalently, $P^{-} z \check{\Gamma}^{*} M_{\theta \mid H^{2}}=\check{\Gamma}^{*} M_{\theta} T_{z}$. Hence $\check{T}_{z} \check{\Gamma}^{*}=\check{\Gamma}^{*} M_{\theta} T_{z} M_{\bar{\theta} \mid \theta H^{2}}$. Finally, $\check{T}_{z} \check{\Gamma}^{*}=$ $\check{\Gamma}^{*} \hat{T}_{z}^{\theta}$, which is the same as $\check{\Gamma}_{\bar{T}}=\hat{T}_{\bar{z}}^{\theta} \check{\Gamma}$. In that case $\check{\Gamma}=\check{\Gamma}_{\varphi}^{\theta}$ where $\theta \bar{\varphi}$ is a 
symbol of the Hankel operator $\left(M_{\bar{\theta}} \check{\Gamma}\right)^{*}=\check{\Gamma} M_{\theta \mid H^{2}}$, so $P^{-}(\theta \bar{\varphi})=\check{\Gamma}^{*} M_{\theta} 1=$ $\check{\Gamma}^{*} \theta$.

At the end of this section we will give formulas using rank-two operators corresponding to relations in Lemma 1.

Proposition 26. Let $\varphi \in L^{\infty}$.

(1) If $\hat{T}=\hat{T}_{\varphi}^{\theta} \in \mathscr{T}\left(\theta H^{2}\right)$, then $\hat{T}-\hat{T}_{z}^{\theta} \hat{T}_{\bar{z}}^{\theta}=(\theta \otimes h+g \otimes \theta)_{\mid \theta H^{2}}$ with $h=\theta \overline{P^{-} \varphi} \in \theta H^{2}, g=\theta P^{+} \varphi \in \theta H^{2}$.

(2) If $\check{T}=\check{T}_{\varphi} \in \mathscr{T}\left(H_{-}^{2}\right)$, then $\check{T}-\check{T}_{\bar{z}} \check{T}_{z}=(\bar{z} \otimes h+g \otimes \bar{z})_{\mid H_{-}^{2}}$ with $h=$ $\bar{z} P^{-} \bar{\varphi} \in H_{-}^{2}, g=P^{-}(\bar{z} \varphi) \in H_{-}^{2}$.

(3) If $\hat{\Gamma}=\hat{\Gamma}_{\varphi}^{\theta} \in \mathscr{T}\left(\theta H^{2}, H_{-}^{2}\right)$, then $\check{T}_{\bar{z}} \hat{\Gamma}-\hat{\Gamma} \hat{T}_{\bar{z}}^{\theta}=(\bar{z} \otimes h+g \otimes \bar{z})_{\mid \theta H^{2}}$ with $h=-P_{\theta H^{2}} \bar{\varphi} \in \theta H^{2}, g=P^{-}\left(C_{\theta} \bar{\varphi}\right) \in H_{-}^{2}$.

(4) If $\check{\Gamma}=\check{\Gamma}_{\varphi}^{\theta} \in \mathscr{T}\left(H_{-}^{2}, \theta H^{2}\right)$, then $\check{\Gamma}_{z}-\hat{T}_{z}^{\theta} \check{\Gamma}=(\theta \otimes h+g \otimes \bar{z})_{\mid H_{-}^{2}}$ with $h=P^{-}\left(C_{\theta} \varphi\right) \in H_{-}^{2}, g=-P_{\theta H^{2}} \varphi \in \theta H^{2}$.

Proof. For the proof of (1) note that by Proposition 20, $\hat{T}_{\varphi}^{\theta}=M_{\theta} T_{\varphi} M_{\bar{\theta} \mid \theta H^{2}}$, and so we have $\hat{T}_{\varphi}^{\theta}-\hat{T}_{z}^{\theta} \hat{T}_{\varphi}^{\theta} \hat{T}_{\bar{z}}^{\theta}=M_{\theta}\left(T_{\varphi}-T_{z} T_{\varphi} T_{\bar{z}}\right) M_{\bar{\theta}}$. Since

$$
T_{\varphi}-T_{z} T_{\varphi} T_{\bar{z}}=1 \otimes \overline{P^{-} \varphi}+P^{+} \varphi \otimes 1,
$$

by Proposition 19 (4) we get

$$
\hat{T}_{\varphi}^{\theta}-\hat{T}_{\bar{z}}^{\theta} \hat{T}_{\varphi}^{\theta} \hat{T}_{z}^{\theta}=\theta \otimes \theta \overline{P^{-} \varphi}+\theta P^{+} \varphi \otimes \theta .
$$

To obtain (2) note that

$$
\begin{aligned}
\check{T}_{\varphi}-\check{T}_{\bar{z}} \check{T}_{\varphi} \check{T}_{z} & =J\left(T_{\bar{\varphi}}-T_{z} T_{\bar{\varphi}} T_{\bar{z}}\right) J=J\left(1 \otimes \overline{P^{-} \bar{\varphi}}+P^{+} \bar{\varphi} \otimes 1\right) J \\
& =\bar{z} \otimes J \overline{P^{-} \bar{\varphi}}+J P^{+} \bar{\varphi} \otimes \bar{z}=\bar{z} \otimes \bar{z} P^{-} \bar{\varphi}+P^{-} J \bar{\varphi} \otimes \bar{z} \\
& =\bar{z} \otimes \bar{z} P^{-} \bar{\varphi}+P^{-}(\bar{z} \varphi) \otimes \bar{z}
\end{aligned}
$$

by Propositions 19 and 20 (5). Now to show (3) take $f \in \theta H^{2}$. Then by Lemma 1 we have

$$
\begin{aligned}
\left(\check{T}_{\bar{z}} \hat{\Gamma}_{\varphi}^{\theta}\right. & \left.-\hat{\Gamma}_{\varphi}^{\theta} \hat{T}_{\bar{z}}^{\theta}\right) f=\bar{z} P^{-} \varphi f-P^{-} \varphi(\bar{z} f-\theta \bar{z}(\bar{\theta} f)(0)) \\
& =\bar{z} P^{-} \varphi f-P^{-} \bar{z} \varphi f+(\bar{\theta} f)(0) P^{-} \theta \bar{z} \varphi=-\langle\varphi f, 1\rangle \bar{z}+\langle\bar{\theta} f, 1\rangle P^{-}\left(C_{\theta} \bar{\varphi}\right) \\
& =-\left\langle f, P_{\theta H^{2}} \bar{\varphi}\right\rangle \bar{z}+\langle f, \theta\rangle P^{-}\left(C_{\theta} \bar{\varphi}\right)=\left(-\bar{z} \otimes P_{\theta H^{2}} \bar{\varphi}+P^{-}\left(C_{\theta} \bar{\varphi}\right) \otimes \theta\right) f
\end{aligned}
$$

using Proposition 20 and Corollary 21 (1). To prove (4) take $f \in H_{-}^{2}$ and note that, by Lemma 1 (2) and Proposition 20, we have

$$
\begin{aligned}
& \left(\check{\Gamma}_{\varphi}^{\theta} \check{T}_{z}-\hat{T}_{z}^{\theta} \check{\Gamma}_{\varphi}^{\theta}\right) f=\theta P^{+} \bar{\theta} \varphi \check{T}_{z} f-z \theta P^{+} \bar{\theta} \varphi f=\theta\left(P^{+} \bar{\theta} \varphi(z f-\langle f, \bar{z}\rangle)-z P^{+} \bar{\theta} \varphi f\right) \\
& \quad=\theta\left(\left(P^{+} z-z P^{+}\right) \bar{\theta} \varphi f-\langle f, \bar{z}\rangle P^{+} \bar{\theta} \varphi\right)=\theta\left(\langle\bar{\theta} \varphi f, \bar{z}\rangle-\langle f, \bar{z}\rangle P^{+}(\bar{\theta} \varphi)\right) \\
& \quad=\left\langle f, C_{\theta} \varphi\right\rangle \theta-\langle f, \bar{z}\rangle \theta P^{+}(\bar{\theta} \varphi)=\left(\theta \otimes P^{-}\left(C_{\theta} \varphi\right)-P_{\theta H^{2}}(\varphi) \otimes \bar{z}\right) f .
\end{aligned}
$$




\section{Characterization of Dual Truncated Toeplitz Operators}

The restrictions of dual truncated Toeplitz operators considered in Sect. 6 will be used to give a necessary and sufficient condition for any operator $D \in$ $\mathscr{B}\left(K_{\theta}^{\perp}\right)$ to be a DTTO. Moreover, we can easily recover its unique symbol.

Theorem 27. Let $\theta$ be an inner function and let $D \in \mathscr{B}\left(K_{\theta}^{\perp}\right)$. Then $D$ is a dual truncated Toeplitz operator, $D \in \mathscr{T}\left(K_{\theta}^{\perp}\right)$, if and only if the following conditions hold

(1) $P_{\theta H^{2}} D_{\mid \theta H^{2}}=\hat{T}_{\bar{z}}^{\theta} P_{\theta H^{2}} D_{\mid \theta H^{2}} \hat{T}_{z}^{\theta}$;

(2) $P^{-} D_{\mid H_{-}^{2}}=C_{\theta} P_{\theta H^{2}} D_{\mid \theta H^{2}}^{*} C_{\theta}$;

(3) $P^{-} D_{\mid \theta H^{2}} \hat{T}_{z}^{\theta}=\check{T}_{z} P^{-} D_{\mid \theta H^{2}}$ and $\left(P_{\theta H^{2}} D_{\mid H_{-}^{2}}\right)^{*} \hat{T}_{z}^{\theta}=\check{T}_{z}\left(P_{\theta H^{2}} D_{\mid H_{-}^{2}}\right)^{*}$;

(4) $P^{-}(D(\theta))=P^{-}\left(\theta^{2} \overline{D^{*}(\theta)}\right)$ and $P^{-}\left(D^{*}(\theta)\right)=P^{-}\left(\theta^{2} \overline{D(\theta)}\right)$.

In that case, $D=D_{\varphi}^{\theta}$ with $\varphi \in L^{\infty}$ given by

$$
\varphi=P^{+}(\bar{\theta} D(\theta))+\overline{P^{+}\left(\bar{\theta} D^{*}(\theta)\right)}-\langle D(\theta), \theta\rangle .
$$

Remark 28. Equality (7.1) gives a formula for the symbol $\varphi$ of a dual truncated Toeplitz operator $D$ by using the value of $D$ and its adjoint $D^{*}$ on the function $\theta$. The symbol $\varphi \in L^{\infty}$ can be equivalently calculated using different formulas. Let $\varphi=\varphi^{-}+\varphi^{+}, \varphi^{-} \in H_{-}^{2}, \varphi^{+} \in H^{2}$ and let $\hat{\varphi}(0)$ denote the 0 -th Fourier coefficient of $\varphi$. Then using Corollary 2 we have

(1) $\hat{\varphi}(0)=\langle D \theta, \theta\rangle=\langle D \bar{z}, \bar{z}\rangle$,

(2) $\varphi^{+}=P^{+} \bar{\theta} D \theta=\bar{\theta} P_{\theta H^{2}}(D \theta)=\overline{z P^{-}\left(D^{*} \bar{z}\right)}$,

(3) $\varphi^{-}=\overline{P^{+}\left(\bar{\theta} D^{*}(\theta)\right)}-\hat{\varphi}(0)=\overline{z P^{+}\left(\bar{z} \bar{\theta} D^{*}(\theta)\right)}=z P^{-}(D(\bar{z}))-\hat{\varphi}(0)=$ $P^{-}(z D(\bar{z}))$.

Proof of Theorem 2\%. Assume firstly that $D=D_{\varphi}^{\theta}$ with $\varphi \in L^{\infty}$. Then

$$
D_{\varphi}^{\theta}=\left[\begin{array}{cc}
\hat{T}_{\varphi}^{\theta} & \check{\Gamma}_{\varphi}^{\theta} \\
\hat{\Gamma}_{\varphi}^{\theta} & \check{T}_{\varphi}
\end{array}\right]=\left[\begin{array}{cc}
\hat{T}_{\varphi}^{\theta} & \left(\hat{\Gamma}_{\varphi}^{\theta}\right)^{*} \\
\hat{\Gamma}_{\varphi}^{\theta} & \check{T}_{\varphi}
\end{array}\right] .
$$

Hence (1) is satisfied by Theorem 25 (a), (2) is satisfied by Proposition 22 and (3) is satisfied by Theorem 25 (c), (d). Moreover,

$$
D(\theta)=P_{\theta}^{\perp}(\varphi \theta)=\theta P^{+}(\varphi)+P^{-}(\varphi \theta)
$$

and

$$
D^{*}(\theta)=P_{\theta}^{\perp}(\bar{\varphi} \theta)=\theta P^{+}(\bar{\varphi})+P^{-}(\bar{\varphi} \theta) .
$$

It follows that $P^{-}(D(\theta))=P^{-}(\varphi \theta)$ and

$$
P^{-}\left(\theta^{2} \overline{D^{*}(\theta)}\right)=P^{-}\left(\theta \overline{P^{+}(\bar{\varphi})}\right)=P^{-}(\varphi \theta)=P^{-}(D(\theta)) .
$$

Similarly, $P^{-}\left(D^{*}(\theta)\right)=P^{-}(\bar{\varphi} \theta)$ and

$$
P^{-}\left(\theta^{2} \overline{D(\theta)}\right)=P^{-}\left(\theta \overline{P^{+}(\varphi)}\right)=P^{-}(\bar{\varphi} \theta)=P^{-}\left(D^{*}(\theta)\right) .
$$

Thus (4) is also satisfied. 
Assume now that $D=\left[\begin{array}{cc}P_{\theta H^{2}} D_{\mid \theta H^{2}} & P_{\theta H^{2}} D_{\mid H_{-}^{2}} \\ P^{-} D_{\mid \theta H^{2}} & P^{-} D_{\mid H_{-}^{2}}\end{array}\right] \in \mathscr{B}\left(K_{\theta}^{\perp}\right)$ satisfies (1)-

(4). It then follows from (1) and Theorem 25 (a) that $P_{\theta H^{2}} D_{\mid \theta H^{2}}=\hat{T}_{\varphi}^{\theta}$ for $\varphi \in L^{\infty}$ given by

$$
\varphi=\bar{\theta} P_{\theta H^{2}} D_{\mid \theta H^{2}}(\theta)+\theta \overline{\left(P_{\theta H^{2}} D_{\mid \theta H^{2}}^{*}(\theta)\right)}-\left\langle P_{\theta H^{2}} D_{\mid \theta H^{2}}(\theta), \theta\right\rangle .
$$

By (2) and Proposition 22,

$$
P^{-} D_{\mid H_{-}^{2}}=C_{\theta} P_{\theta H^{2}} D_{\mid \theta H^{2}}^{*} C_{\theta}=C_{\theta} \hat{T}_{\bar{\varphi}}^{\theta} C_{\theta}=\check{T}_{\varphi} .
$$

By (3) and Theorem 25 (c), (d) there exist $\psi, \chi \in L^{\infty}$ such that $P^{-} D_{\mid \theta H^{2}}=\hat{\Gamma}_{\psi}^{\theta}$ with $P^{-}(\theta \psi)=P^{-} D_{\mid \theta H^{2}}(\theta)$ and similarly $\left(P_{\theta H^{2}} D_{\mid H_{-}^{2}}\right)^{*}=\hat{\Gamma}_{\chi}^{\theta}$ with $P^{-}(\theta \chi)=$ $\left(P_{\theta H^{2}} D_{\mid H_{-}^{2}}\right)^{*}(\theta)$. We will now use (4) to show that

$$
(\varphi-\psi) \perp \overline{\theta z H^{2}} \text { and } \quad(\bar{\varphi}-\chi) \perp \overline{\theta z H^{2}} .
$$

Since $\varphi$ is given by (7.2), using the first equality in (4) we get

$$
\begin{aligned}
P^{-}(\theta \varphi) & =P^{-}\left(\theta^{2} \overline{\left(P_{\theta H^{2}} D_{\mid \theta H^{2}}^{*}(\theta)\right)}\right)=P^{-}\left(\theta^{2} \overline{D^{*}(\theta)}\right) \\
& =P^{-}(D(\theta))=P^{-} D_{\mid \theta H^{2}}(\theta)=P^{-}(\theta \psi),
\end{aligned}
$$

and so $(\theta \varphi-\theta \psi) \perp H_{-}^{2}$. Similarly, using the second equality in (4) we get

$$
\begin{aligned}
P^{-}(\theta \bar{\varphi}) & =P^{-}\left(\theta^{2} \overline{\left(P_{\theta H^{2}} D_{\mid \theta H^{2}}(\theta)\right)}\right)=P^{-}\left(\theta^{2} \overline{D(\theta)}\right) \\
& =P^{-}\left(D^{*}(\theta)\right)=\left(P_{\theta H^{2}} D_{\mid H_{-}^{2}}\right)^{*}(\theta)=P^{-}(\theta \chi),
\end{aligned}
$$

hence $(\theta \bar{\varphi}-\theta \chi) \perp H_{-}^{2}$. Thus, by (6.1), we proved that $P^{-} D_{\mid \theta H^{2}}=\hat{\Gamma}_{\varphi}^{\theta}$ and $\left(P_{\theta H^{2}} D_{\mid H_{-}^{2}}\right)^{*}=\hat{\Gamma}_{\bar{\varphi}}^{\theta}$, that is, $P_{\theta H^{2}} D_{\mid H_{-}^{2}}=\left(\hat{\Gamma}_{\bar{\varphi}}^{\theta}\right)^{*}=\check{\Gamma}_{\varphi}^{\theta}$. Therefore,

$$
D_{\varphi}^{\theta}=\left[\begin{array}{cc}
\hat{T}_{\varphi}^{\theta} & \check{\Gamma}_{\varphi}^{\theta} \\
\hat{\Gamma}_{\varphi}^{\theta} & \check{T}_{\varphi}
\end{array}\right]
$$

Moreover,

$$
\begin{aligned}
\varphi & =P^{+}\left(\bar{\theta}\left(P_{\theta H^{2}} D_{\mid \theta H^{2}}(\theta)\right)\right)+\overline{P^{+}\left(\bar{\theta} P_{\theta H^{2}} D_{\mid \theta H^{2}}^{*}(\theta)\right)}-\left\langle P_{\theta H^{2}} D_{\mid \theta H^{2}}(\theta), \theta\right\rangle \\
& =P^{+}(\bar{\theta} D(\theta))+\overline{P^{+}\left(\bar{\theta} D^{*}(\theta)\right)}-\langle D(\theta), \theta\rangle .
\end{aligned}
$$

Example 29. Let $\theta(z)=z^{n}$, take $D \in \mathscr{B}\left(K_{\theta}^{\perp}\right)$ and denote by $\left[a_{i, j}\right]$ the matrix of $D$ with respect to the monomial basis $\left\{z^{k}: k \geq n \vee k<0\right\}$. Then the conditions in (4) can be written as

$$
a_{-k, n}=a_{n, k+2 n} \quad \text { and } \quad a_{n,-k}=a_{k+2 n, n} \text { for all } k>0 .
$$

\section{Compliance with ethical standards}

Conflict of interest The authors declare that they have no conflict of interest. 
Open Access. This article is licensed under a Creative Commons Attribution 4.0 International License, which permits use, sharing, adaptation, distribution and reproduction in any medium or format, as long as you give appropriate credit to the original author(s) and the source, provide a link to the Creative Commons licence, and indicate if changes were made. The images or other third party material in this article are included in the article's Creative Commons licence, unless indicated otherwise in a credit line to the material. If material is not included in the article's Creative Commons licence and your intended use is not permitted by statutory regulation or exceeds the permitted use, you will need to obtain permission directly from the copyright holder. To view a copy of this licence, visit http://creativecommons. org/licenses/by/4.0/.

\section{References}

[1] Baranov, A., Chalendar, I., Fricain, E., Mashreghi, J.E., Timotin, D.: Bounded symbols and reproducing kernel thesis for truncated Toeplitz operators. J. Funct. Anal. 259, 2673-2701 (2010)

[2] Bendaoud, Z., Saouli, N.: On the product of dilation of truncated Toeplitz operators. Turk. J. Math. 44, 142-151 (2020)

[3] Bessonov, R.V.: Fredholmness and compactness of truncated Toeplitz and Hankel operators. Integr. Equ. Oper. Theory 82, 451-467 (2015)

[4] Câmara, M.C., Carteiro, C.: Toeplitz kernels and finite rank truncated Toeplitz operators. Recent trends in operator theory and applications, 43-62, Contemp. Math., 737, Amer. Math. Soc., Providence, RI (2019)

[5] Câmara, M.C., Kliś-Garlicka, K., Łanucha, B., Ptak, M.: Conjugations in $L^{2}$ and their invariants. Anal. Math. Phys. 10, 22 (2020). https://doi.org/10.1007/ s13324-020-00364-5

[6] Câmara, M.C., Kliś-Garlicka, K., Łanucha, B., Ptak, M.: Invertibility, Fredholmness and kernels of dual truncated Toeplitz operators. Banach J. Math. Anal. 14, 1558-1580 (2020). https://doi.org/10.1007/s43037-020-00077-8

[7] Câmara, M.C., Ross, W.T.: The dual of the compressed shift. Can. Math. Bull. (2020). https://doi.org/10.4153/S0008439520000260

[8] Ding, X., Sang, Y.: Dual truncated Toeplitz operators. J. Math. Anal. Appl. 461, 929-946 (2018)

[9] Duren, P.L.: Theory of $H^{p}$ Spaces, vol. 38. Academic Press, New York (1970)

[10] Fricain, E., Hartmann, A., Ross, W.T.: Multipliers between model spaces. Stud. Math. 240, 177-191 (2018)

[11] Garcia, S.R., Mashreghi, J.E., Ross, W.: Introduction to Model Spaces and their Operators, Cambridge Studies in Advanced Mathematics, 148. Cambridge University Press, Cambridge (2016)

[12] Garcia, S.R., Putinar, M.: Complex symmetric operators and applications. Trans. Am. Math. Soc. 358, 1285-1315 (2006)

[13] Garcia, S., Ross, W.T.: Model spaces: a survey. Invariant subspaces of the shift operator, 197-245, Contemp. Math. 638, Amer. Math. Soc., Providence, RI (2015) 
[14] Gu, C.: Algebraic properties of truncated Hankel operators, preprint

[15] Hu, Y., Deng, J., Yu, T., Liu, L., Lu, Y.: Reducing Subspaces of the Dual Truncated Toeplitz Operator. J. Funct. Spaces, Art. ID 7058401, p 9 (2018)

[16] Koosis, P.: Introduction to $H^{p}$ Spaces, 2nd edn. Cambridge University Press, Cambridge (1998)

[17] Ma, P., Yan, F., Zhang, D.: Zero, finite rank, and compact big truncated Hankel operators on model spaces. Proc. Am. Math. Soc. 146, 5235-5242 (2018)

[18] Peller, V.V.: Hankel Operators and Their Applications. Springer, New York (2003)

[19] Ptak, M., Simik, K., Wicher, A.: C-normal operators. Electron. J. Linear Algebra 36, 67-79 (2020)

[20] Sang, Y., Qin, Y., Ding, X.: Dual truncated Toeplitz $C^{*}$-algebras. Banach J. Math. Anal. 13(2), 275-292 (2019)

[21] Sang, Y., Qin, Y., Ding, X.: A theorem of Brown-Halmos type for dual truncated Toeplitz operators. Ann. Funct. Anal. 11(2), 271-284 (2020)

[22] Sarason, D.: Algebraic properties of truncated Toeplitz operators. Oper. Matrices 1, 491-526 (2007)

[23] Sarason, D.: Generalized interpolation in $H^{\infty}$. Trans. Am. Math. Soc. 127, 179203 (1967)

\section{Cristina Câmara}

Center for Mathematical Analysis, Geometry and Dynamical Systems, Mathematics Department, Instituto Superior Técnico

Universidade de Lisboa

Av. Rovisco Pais

1049-001 Lisboa

Portugal

e-mail: ccamara@math.ist.utl.pt

Kamila Kliś-Garlicka and Marek Ptak

Department of Applied Mathematics

University of Agriculture

ul. Balicka 253c

30-198 Kraków

Poland

e-mail: rmklis@cyfronet.pl;

rmptak@cyf-kr.edu.pl

Bartosz Łanucha

Department of Mathematics

Maria Curie-Skłodowska University

pl. M. Curie-Skłodowskiej 1

20-031 Lublin

Poland

e-mail: bartosz.lanucha@poczta.umcs.lublin.pl 
Received: May 24, 2020.

Accepted: September 17, 2020.

Publisher's Note Springer Nature remains neutral with regard to jurisdictional claims in published maps and institutional affiliations. 